\title{
MeCP2 Is Critical within HoxB1-Derived Tissues of Mice for Normal Lifespan
}

\author{
Christopher S. Ward,,$^{1,2}$ E. Melissa Arvide, ${ }^{1,3}$ Teng-Wei Huang, ${ }^{1,4}$ Jong Yoo, ${ }^{5}$ Jeffrey L. Noebels, ${ }^{2,5,6}$ \\ and Jeffrey L. Neul ${ }^{1,2,3,4,6,7,8}$ \\ ${ }^{1}$ Jan and Dan Duncan Neurological Research Institute, Houston, Texas 77030, and ${ }^{2}$ Department of Molecular and Human Genetics, ${ }^{3}$ Department of \\ Pediatrics, ${ }^{4}$ Program in Developmental Biology, ${ }^{5}$ Developmental Neurogenetics Laboratory, Department of Neurology, ${ }^{6}$ Department of Neuroscience, \\ ${ }^{7}$ Program in Translational Biology and Molecular Medicine, and ${ }^{8}$ Department of Molecular Physiology and Biophysics, Baylor College of Medicine, \\ Houston, Texas 77030
}

Rett syndrome is a neurodevelopmental disorder caused by mutations in methyl-CpG-binding protein 2 (MECP2), a transcriptional regulator. In addition to cognitive, communication, and motor problems, affected individuals have abnormalities in autonomic function and respiratory control that may contribute to premature lethality. Mice lacking Mecp2 die early and recapitulate the autonomic and respiratory phenotypes seen in humans. The association of autonomic and respiratory deficits with premature death suggests that $M e c p 2$ is critical within autonomic and respiratory control centers for survival. To test this, we compared the autonomic and respiratory phenotypes of mice with a null allele of Mecp2 to mice with Mecp2 removed from their brainstem and spinal cord. We found that MeCP2 is necessary within the brainstem and spinal cord for normal lifespan, normal control of heart rate, and respiratory response to hypoxia. Restoration of MeCP2 in a subset of the cells in this same region is sufficient to rescue abnormal heart rate and abnormal respiratory response to hypoxia. Furthermore, restoring MeCP2 function in neural centers critical for autonomic and respiratory function alleviates the lethality associated with loss of MeCP2 function, supporting the notion of targeted therapy toward treating Rett syndrome.

\section{Introduction}

Mutations in the X-linked transcriptional regulator methylCpG-binding protein 2 (MECP2) are common in individuals with Rett syndrome (RTT, MIM 312750) (Amir et al., 1999). This disorder predominantly affects girls and is characterized by loss of spoken language and loss of hand skills (Neul et al., 2010). A critical concern in RTT is the incidence of sudden unexpected death, which causes $25 \%$ of all deaths (Kerr et al., 1997). Although the exact cause of such deaths is unknown, breathing abnormalities (Julu et al., 2001; Weese-Mayer et al., 2006; Rohdin et al., 2007) and cardiac rhythm irregularities (Guideri et al.,

Received Jan. 4, 2011; revised May 31, 2011; accepted June 2, 2011.

Author contributions: J. L. Neul designed research; C.S.W., E.M.A., T.-W.H., J.Y., J. L. Noebels, and J. L. Neul performed research; C.S.W., E.M.A., J.Y., J. L. Noebels, and J. L. Neul analyzed data; C.S.W. and J. L. Neul wrote the paper.

This work was supported by the Cynthia and Anthony Petrello Scholar fund at the Jan and Dan Duncan Neurological Research Institute, Texas Children's Hospital (J. L. Neul), U.S. National Institutes of Health Grants NS52240 (J.L. Neul), HD062553 (J.L. Neul), NS066601 (C.S.W.), NS29709 (J. L. Noebels), and HD24064 (Baylor Intellectual and Developmental Disabilities Research (enter), and the International Rett Syndrome Foundation. We thank Huda Zoghbi, Rodney Samaco, Adriano Flora, Alexander Bassuk, Stephen Maricich, Tiemo Klisch, John Fryer, and Benjamin Arenkiel for useful scientific discussions and advice. We thank Sean Cullen, Michael McQueen, Sarah-Ann Pike, and Ekaette Shammah for technical assistance, Corinne Spencer and Richard Paylor and the Neurobehavioral Core for advice on behavioral experiments, Corry Reynolds and the Baylor Mouse Phenotyping Core for assistance and equipment for the plethysmography experiments, and Hui-Chen Lu for access to equipment for stereology experiments. The project described was supported by the Eunice Kennedy Shriver National Institute of Child Health and Human Development (EKS NICHD). The content is solely the responsibility of the authors and does not necessarily represent the official views of the EKS NICHD or the National Institutes of Health.

The authors declare no competing financial interests.

Correspondence should be addressed to Jeffrey L. Neul, Jan and Dan Duncan Neurological Research Institute, 1250 Moursund Street, Suite 1250, Houston, TX 77030. E-mail: jneul@bcm.edu.

DOI:10.1523/JNEUROSCI.0057-11.2011

Copyright $\odot 2011$ the authors $\quad 0270-6474 / 11 / 3110359-12 \$ 15.00 / 0$
1999; Madan et al., 2004) are suspected to be causative. Characterization of breathing and heart rate revealed a lack of coordination between the two, suggesting alteration in the medullary network that integrates these systems (Julu et al., 2001). Most boys with MECP2 mutations are severely affected with congenital encephalopathy and death within 3 years of life associated with respiratory arrest (Kankirawatana et al., 2006) and marked bradycardia (Schüle et al., 2008) at death.

Mice lacking MeCP2 function reproduce features of the human disorder (Chen et al., 2001; Guy et al., 2001). Male animals with a null allele of Mecp2 (NULL) develop severe neurological abnormalities and breathing irregularities (Viemari et al., 2005; Ogier et al., 2007; Roux et al., 2008; Zanella et al., 2008), and die between 8 and 12 weeks of life (Guy et al., 2001). Heterozygous female mice lacking one copy of Mecp2 develop similar phenotypes in a delayed and variable manner, likely due to variable patterns of $\mathrm{X}$-chromosome inactivation in individual animals (Young and Zoghbi, 2004). Because this variability compounds experimental complexity, most research has been performed using male NULL mice.

Surprisingly, restoring $\mathrm{MeCP} 2$ function in all cells can reverse abnormalities in mice (Guy et al., 2007), demonstrating that loss of MeCP2 does not cause irreversible changes in cellular function and providing hope that the human disorder may be reversible. However, restoring function only to the forebrain did not rescue lethality (Alvarez-Saavedra et al., 2007), suggesting that the critical region for MeCP2 function is caudal to the forebrain.

One hypothesis is that specific clinical features present in RTT are the cumulative result of loss of $\mathrm{MeCP} 2$ function within subsets of cells. A variety of reports support the hypothesis that dis- 
tinct phenotypes found in the NULL animals can be reproduced by removing MeCP2 in specific cell populations (Fyffe et al., 2008; Adachi et al., 2009; Samaco et al., 2009; Chao et al., 2010). Because the hindbrain includes neuronal circuits critical for the regulation of autonomic and respiratory function that may contribute to lethality, we hypothesized that the autonomic and respiratory dysfunction as well as the premature death seen in NULL animals is due to $\mathrm{MeCP} 2$ requirements within the hindbrain. To test this hypothesis, we either removed or restored MeCP2 function within the medulla and spinal cord. From this, we show that MeCP2 is both necessary and sufficient within this region for a number of physiological processes and normal lifespan.

\section{Materials and Methods}

Animals used in experiments. All research and animal care procedures were approved by the Baylor College of Medicine Institutional Animal Care and Use Committee and housed in the Association for Assessment and Accreditation of Laboratory Animal Care-approved animal facility at Baylor College of Medicine. Mecp $2^{\text {Tm1Bird }}$ mice (Guy et al., 2001) were obtained as a gift from Dr. Adrian Bird (University of Edinburgh, Edinburgh, UK) and backcrossed and maintained on a 129S6 background for $>10$ generations. HPRT-Cre mice (Su et al., 2002) were crossed to the Mecp $2^{\text {Tm1Bird }}$ mice to generate Mecp $2^{\text {Tm1.1Bird }}$ NULL mice. Mecp $2^{\text {Tm2Bird }}$ mice (Guy et al., 2007) were obtained from The Jackson Laboratory and maintained on a C57BL/6J background. Mecp $2^{\text {TM1Jae }}$ (Chen et al., 2001) animals were acquired from the University of California-Davis Mouse Mutant Resource Center and maintained on a C56BL/6J strain. HoxB ${ }^{\text {cre }}$ mice (Arenkiel et al., 2003) were maintained on a C57BL/6 background, and crossed to Mecp $2^{\text {Tm1Bird }}$, Mecp $2^{\text {Tm2Bird }}$, or Mecp $2^{\text {TM1Jae }}$ mice to generate male experimental animals. Identification of adult structures derived from the HoxB1 lineage was facilitated by using mice with an allele of $n$ LacZ-IRES-myrGFP expressed under control of the Tau promoter, or an allele of LacZ integrated into the Rosa26 locus (Soriano, 1999; Hippenmeyer et al., 2005).

Western blot detection of proteins. MeCP2 expression was determined by Western blot from brain tissue. Fresh brains were dissected from male mice and processed as hemibrains or further dissected to cerebrum and brainstem. The brains were homogenized in $100 \mathrm{~mm}$ Tris, $\mathrm{pH} 8.0$, and 2\% SDS via Dounce homogenizer with the B handle and rocked at room temperature for at least $1 \mathrm{~h}$ to ensure complete cell lysis. Soluble proteins were separated from cell debris by centrifugation at 20,000 $\times g$ for 15 $\mathrm{min}$, and the supernatant was then loaded onto a PAGE gel and transferred onto a nitrocellulose membrane. The membrane was blocked in Tris-buffered saline, pH 7.6, and $0.1 \%$ Tween 20 (TBS-T) with 5\% milk. For immunodetection of MeCP2, an antibody raised in rabbit (Shahbazian et al., 2002) diluted 1:2000 was used; detection of the loading control GAPDH was performed with an antibody raised in mouse (Millipore) diluted 1:20,000. HRP-conjugated secondary antibodies against mouse and rabbit were used at a dilution of 1:4000 (GE) and visualized with ECL detection reagent (GE), or Dura substrate (Pierce). The signals were captured on film and scanned or captured with a blot imager (Alpha Inotech) and quantified by densitometry with ImageJ software (Rasband, 1997-2011).

Glucose tolerance testing. Male mice were fasted overnight for $16 \mathrm{~h}$ and then given an acute dose of glucose at $2 \mathrm{~g}$ (glucose) $/ \mathrm{kg}$ (body weight) delivered as $300 \mathrm{mg} / \mathrm{ml}$ glucose prepared in normal saline. Blood glucose measurements were taken by tail bleeds of $\sim 10 \mu$ l per sample and analyzed with a OneTouch Ultramini blood glucometer. Samples were taken immediately before the glucose challenge and at 15,30,60, and $120 \mathrm{~min}$ after the glucose injection.

Chronic electroencephalographic recordings. Male mice (aged 1.5 months) were anesthetized with Avertin (1.25\% tribromoethanol/amyl alcohol solution, i.p.) using a dose of $0.02 \mathrm{ml} / \mathrm{g}$. Teflon-coated silver wire electrodes ( 0.005 inch diameter) soldered to a microminiature connector were implanted bilaterally into the subdural space over frontal, central, parietal, and occipital cortices. Digital electroencephalographic (EEG) activity was monitored daily for up to 2 weeks during prolonged and random $2 \mathrm{~h}$ sample recordings (Stellate Systems, Harmonie software version 5.0b). A digital video camera was used to simultaneously monitor behavior during the EEG recording periods. All recordings were performed at least $24 \mathrm{~h}$ after surgery on mice freely moving in the test cage.

Pulse oximetry. Heart rate data were collected by pulse oximetry with the Mouse Ox (Starr Life Sciences). Measurements were collected from either the tail or neck of male mice. For tail measurements, animals were placed within a restraint tube allowing access to the tail. For neck measurements, fur was removed around the neck with a depilatory cream. The mice were recorded for $5 \mathrm{~min}$ and their heart rate defined as the average over the $5 \mathrm{~min}$ recording. Heart rate data were collected 1-3 times per week throughout the lifespan of the animals. Analysis revealed stable heart rate throughout adulthood, aside from the time period 1 week preceding death, so the values were averaged for the individual animals over the course of their life, excluding the $7 \mathrm{~d}$ before the animal died spontaneously or became moribund and was killed, and then genotype averages were compared.

Measurement of body temperature. Measurement of body temperature was performed in male mice with temperature monitoring implantable radio-frequency transponders (BMDS). The transponders were implanted subcutaneously such that the transponders would reside near the base of the neck. Temperature data were collected at least twice a week between 9:00 A.M. and 12:00 P.M. via a handheld transponder reader. Similar to the heart rate analysis, no change in temperature was observed over the lifespan of the animals except for the $7 \mathrm{~d}$ preceding death. Therefore, the values excluding the $7 \mathrm{~d}$ before death were averaged for each animal, and then genotype averages were compared.

Unrestrained whole-body plethysmography. Male mice were placed within unrestrained whole-body plethysmography chambers (Buxco), $\sim 500 \mathrm{ml}$ in volume with a continuous flow rate of $1 \mathrm{~L} / \mathrm{min}$ flushing the chambers with fresh air. Mice were allowed to acclimate for $20 \mathrm{~min}$, and baseline breathing was then recorded for $30 \mathrm{~min}$. To determine response to hypoxic gas $\left(10 \% \mathrm{O}_{2}\right.$, balance $\left.\mathrm{N}_{2}\right)$, the chamber was then flushed with hypoxic gas for $4 \mathrm{~min}$ and then breathing was recorded during the next 5 min of hypoxic exposure. Breath waveforms and derived parameters, including the instantaneous breathing rate, tidal volume, inspiratory time, and expiratory time, were identified and calculated with Biosystem XA software (Buxco). To reduce the artifacts from excessive movement and sniffing behavior, breaths that exhibited an inspiratory time $<0.03 \mathrm{~s}$, an expiratory time $>10 \mathrm{~s}$, and a calculated exhaled tidal volume $>150 \%$ or $<50 \%$ of calculated inhaled tidal volume were excluded; the recording was then split into $1 \mathrm{~min}$ intervals and only those minutes during which the animal spent $<10 \%$ of its breaths above 500 breaths per minute were included in the analysis (results obtained by this method agreed closely with behavioral monitoring to exclude activity artifacts in a pilot experiment). Plethysmography data were collected in mice between 4 and 21 weeks of age with no significant effect of age on respiratory rate observed nor on tidal volume compared to age-matched controls with the exception of recordings performed immediately preceding death; therefore, values recorded within the last week of life were excluded. Breathing parameters for each animal during baseline and hypoxic challenge were determined as the average instantaneous value over the recorded interval and then averaged across trials. Respiratory parameters were then compared across genotypes.

Immunohistology. Male mice were deeply anesthetized by intraperitoneal injection with Avertin at a dose of $0.04 \mathrm{ml} / \mathrm{g}$ and then fixed by transcardiac perfusion with PBS followed by $4 \%$ paraformaldehyde in PBS. Tissues for histological analysis were harvested: brains were excised from the skull, and the carotid body and superior cervical ganglion were dissected as preps attached to the carotid artery at the level of the carotid bifurcation into the internal and external carotid arteries. The tissues were then fixed overnight in $4 \%$ paraformaldehyde in PBS and cryopreserved by overnight incubations in increasing concentrations of sucrose (up to $30 \%$ sucrose). Tissues were embedded in O.C.T. compound (Sakura) and sectioned. For brain samples, $50 \mu \mathrm{m}$ sections were collected with a cryostat and stained as floating sections. For carotid body and superior cervical ganglion samples, $25 \mu \mathrm{m}$ sections were collected with a cryostat and stained as mounted sections on polylysine-coated slides. Sections were blocked for $1 \mathrm{~h}$ in a PBS solution containing $10 \%$ serum 
(matched to the host used for the secondary antibodies) and $0.3 \%$ Triton $\mathrm{X}-100$. Primary and secondary antibody incubation was performed in the blocking solution for $48 \mathrm{~h}$ at $4^{\circ} \mathrm{C}$ for floating sections and $24 \mathrm{~h}$ at room temperature in a humidity chamber for slide-mounted sections. Sections were washed between incubations with PBS and 0.05\% Triton $\mathrm{X}-100$. If not included in the mounting medium, DAPI and Toto- 3 were included in the penultimate wash. Primary antibodies were used at the following dilutions: rabbit anti-MeCP2 1:250 (Millipore), chicken antiMeCP2 1:500 (Prosci), chicken anti-tyrosine hydroxylase 1:1000 (Abcam), chicken anti- $\beta$-galactosidase 1:1000 (Abcam), rabbit anti-NK1R 1:1000 (Sigma), rabbit anti-somatostatin 1:500 (Immunostar), goat antivesicular acetylcholine transporter 1:1000 (Millipore), and goat antiIslet-1 1:500 (Neuromics). Secondary antibodies conjugated to DyLight 488,549 , or 649 were used at a dilution of 1:500 and raised in goat or donkey (Jackson ImmunoResearch). Sections were mounted with Prolong mounting medium (Invitrogen) and imaged via epifluorescent microscopy (Zeiss M1 with Axiovision software) or laser scanning confocal imaging (Zeiss 710 with ZEN software). Stereological quantification of cells expressing MeCP2 in the hindbrain was performed on coronal sections through the pons and medulla with systematic random sampling of every 12th section, with optical fractionators measuring $144.72 \mu \mathrm{m} \times$ $144.72 \mu \mathrm{m}$ set at an area sampling fraction of 1/64 through the full thickness of the tissue excluding the top plane of focus (West et al., 1991). The pons was defined as ventral to the aqueduct in sections caudal to the substantia nigra through sections containing the locus ceruleus (LC). The medulla was defined as the first section caudal to the locus ceruleus through to the beginning of the spinal cord. Quantification of tyrosine hydroxylase (TH)-positive cells expressing MeCP2 was performed by examining the full cross-sectional area of every eighth section, excluding cells present in the top plane of focus to avoid overestimation of cell numbers, and first identifying cells expressing TH followed by checking for MeCP2 expression. TH-expressing cells were divided into A1, A2, A5, and LC populations based on anatomical location (Paxinos and Franklin, 2001). The nucleus of the solitary tract (NTS) was identified by somatostatin expression in the dorsal medulla (Finley et al., 1981); MeCP2expressing cells were estimated using the optical fractionator technique sampling every 12th slide with a sampling grid area of $22,500 \mu \mathrm{m}^{2}$, dissector height of $15 \mu \mathrm{m}$, and dissector area of $900 \mu \mathrm{m}^{2}$. The retrotrapezoidal nucleus (RTN) was defined as the region ventral to staining for VAchT or ISL1 as a marker of the facial nucleus (Lazarenko et al., 2009); MeCP2-expressing cells were estimated using the optical fractionator technique sampling every fourth slide with a sampling grid area of 22,500 $\mu \mathrm{m}^{2}$, dissector height of $15 \mu \mathrm{m}$, and dissector area of $5625 \mu \mathrm{m}^{2}$. The pre-Botzinger was defined as the somatostatin- or NK1R-stained region ventral to staining for VAchT or ISL1 as a marker of the nucleus ambiguus (Stornetta et al., 2003; Rose et al., 2009); MeCP2-expressing cells were estimated using the optical fractionator technique sampling every fourth slide with a sampling grid area of $8100 \mu \mathrm{m}^{2}$, dissector height of 15 $\mu \mathrm{m}$, and dissector area of $2025 \mu \mathrm{m}^{2}$. The carotid body and superior cervical ganglion were identified by the presence of TH-stained cells at the bifurcation of the carotid artery, and rostral and deep to the carotid artery, respectively (Roux et al., 2008). Quantification was performed using ImageJ with the LOCI Bioformats plug-in (Rasband, 1997-2011) or Stereo Investigator software (MBF Bioscience).

Behavioral characterization. Motor tasks (dowel walking, wire hang, footslip, accelerating rotarod), social interaction (partition test), learning (conditioned fear), anxiety (elevated plus maze, light-dark), and openfield analysis were performed on male mice as previously described (Samaco et al., 2008).

Statistics. All statistics were performed using SPSS v.12 on a PC. Parametric statistics were performed using ANOVA with genotype as a factor. For conditions in which the genotype factor had more than two levels, formalized post hoc testing was performed using Tukey's HSD correction to detect pairwise differences. Survival analysis was performed using Kaplan-Meier survival analysis, with Tarone-Ware method applied to detect differences in survival between genotype groups.

\section{Results}

Removal of MeCP2 from the HoxB1 domain targets the hindbrain and several key respiratory and autonomic centers To remove $\mathrm{MeCP} 2$ from the medulla, we bred mice that contain a conditional Mecp2 allele flanked by loxP sequences $\left(M e c p 2^{f l o x /+}\right)$ (Guy et al., 2001) to mice that express the Cre recombinase in the HoxB1 domain $\left(H o x B 1^{\text {cre }}\right.$ ) (Arenkiel et al., 2003). HoxB1 ${ }^{\text {cre }}$ drives Cre expression in the rhombencephalon distal to the posterior border of rhombomere 3, which encompasses the caudal part of the pons, the medulla, and the spinal cord. To verify that MeCP2 protein expression was disrupted specifically within the hindbrain of $H o x B 1^{\text {cre/WT}} ; M e c p 2^{\text {flox/Y }}$ (hereafter referred to as CKO for "conditional knock-out") animals, we performed Western blotting on both the hindbrain and forebrain tissues (Fig. 1A), including samples from other genotypes $\left(H o x B 1^{W T / W T}\right.$; Mecp $2^{W T / Y}$ referred to as "WT," HoxB1 $1^{\text {cre/WT}}$; $M e c p 2^{W T / Y}$ referred to as "CRE," and HoxB1 $1^{W T / W T} ; M e c p 2^{\text {flox/Y }}$ referred to as "FLOX") as littermate controls. MeCP2 protein was absent from the hindbrain of $\mathrm{CKO}$ animals but present in the forebrain. In contrast, $\mathrm{MeCP} 2$ was present in both brain regions of the control genotypes. The ratio of $\mathrm{MeCP} 2$ in the hindbrain to that in the forebrain was dramatically reduced in the $\mathrm{CKO}$ animals compared to all other genotypes (Fig. $1 B$ ).

To further evaluate the regions targeted by the $H o x B 1^{\text {cre }}$, we bred the $H o x B 1^{\text {cre }}$ mice with reporter mice either possessing an allele of $n L a c Z$-IRES-myrGFP expressed under control of the Tau promoter or a conditional $\beta$-galactosidase reporter integrated into the ROSA26 locus (Soriano, 1999; Hippenmeyer et al., $2005)$. Both of these reporter alleles express $\beta$-galactosidase in a Cre-dependent fashion, one in all tissues and one only in neurons. Within the brain, the rostral boundary of the HoxB1 domain lies within the pons (Fig. $1 C$ ). Due to curvature of the neuraxis, the dorsal pons represents more rostrally defined structures and is excluded from the HoxB1 domain (Fig. 1D). However, the ventral pons is derived from a more caudal origin and is within the HoxB1 domain. In contrast, the medulla was completely derived from the HoxB1 domain (Fig. 1E). Within the hindbrain, we investigated several subpopulations of neurons involved in autonomic and breathing control to ensure that they were targeted by $\mathrm{HoxB1}^{\text {cre }}$. The A5, A2, and A1 noradrenergic neurons are all targeted by $H o x B 1^{\text {cre }}$ (Fig. $1 F, H, I$ ), while the LC in the dorsal pons is not (Fig. $1 G$ ). Key respiratory centers of the ventral medulla such as the retrotrapezoid nucleus and preBotzinger complex are also included within the HoxB1 domain (Fig. $1 \mathrm{~J}, K$ ). The peripherally located carotid body, which is critical for recognizing blood oxygenation to modulate respiratory function, is also targeted by $\mathrm{HoxB1}^{\text {cre }}$ (Fig. $1 L, M$ ). Together, these data show that the $H o x B 1^{\text {cre }}$ targets several regions critical for regulation of respiratory and autonomic function, but excludes some such as the locus ceruleus.

\section{Removing MeCP2 from the HoxB1 domain impairs motor coordination but does not modify forebrain-associated behaviors}

Male mice with a null allele of Mecp2 exhibit a variety of movement problems (Chen et al., 2001; Guy et al., 2001; Pratte et al., 2011). Our CKO animals performed poorly on a number of motor coordination tasks compared to control genotypes, including dowel rod walking (Fig. 2A) and wire hanging (Fig. 2 B). Moreover, $\mathrm{CKO}$ animals were not able to walk well on a metal grid, having more footslips than all control genotypes (Fig. 2C). CKO animals also performed poorly on the accelerating rotating rod (Fig. 2D). 
A

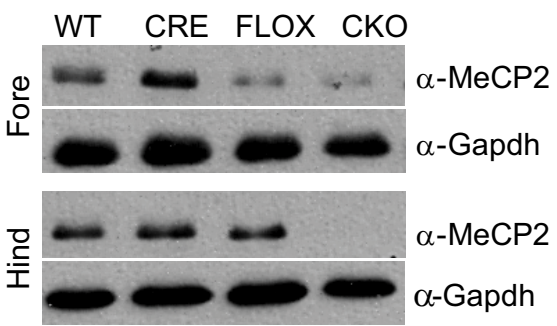

B

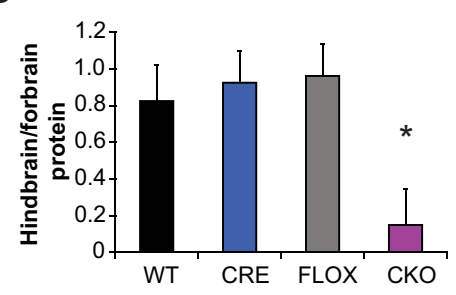

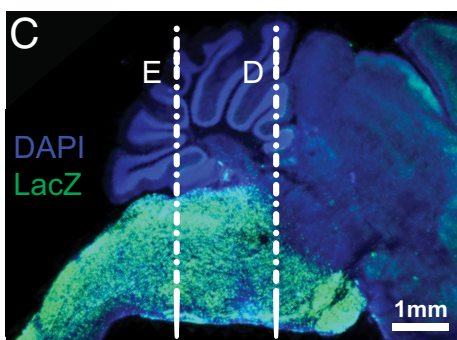
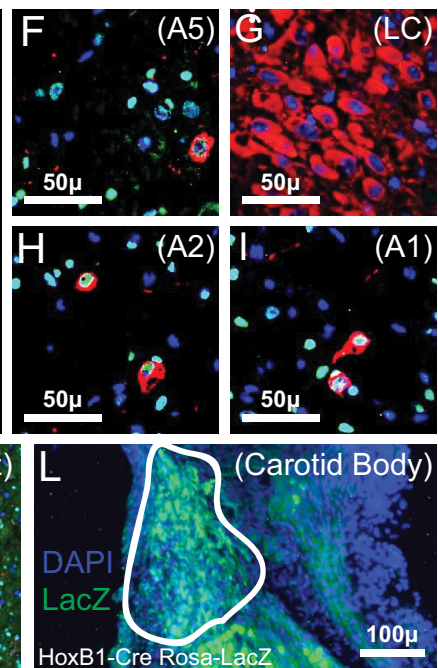

$100 \mu$

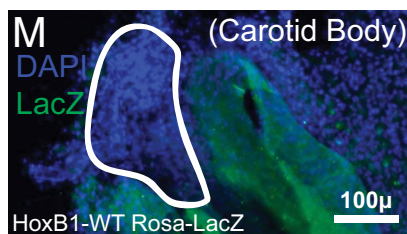

Figure 1. HoxB1-Cre targets tissues that give rise to the hindbrain. MeCP2 protein level was reduced in the hindbrain of $C K 0$ animals but unchanged in the forebrain compared with the forebrain ofFLOX animals as detected by Western blot $(\boldsymbol{A})$. Quantification showed a decrease in the ratio of hindbrain to forebrain MeCP2 protein for CKO animals $\left(\boldsymbol{B}, n=4\right.$ for each genotype, ${ }^{*} p<0.05,1$-way ANOVA with genotype as a factor and $p<0.05$, post hoc pairwise difference between (KO and all other genotypes assessed using Tukey HSD post hoc testing; error bars indicate SEM). HoxB1-Cre is expressed in tissues that give rise to the hindbrain and spinal cord as revealed by staining for a (re-dependent LacZ reporter (green) in a midsagittal section through the brain (C). The dotted lines in C are approximate positions of the coronal sections in $\boldsymbol{D}$ and $\boldsymbol{E}$ also taken from animals possessing a lacZ reporter activated by HoxB1-Cre. The rostral boundary of HoxB1 defined tissues in the adult lies within the pons, with the rostrally defined dorsal pons being excluded from the HoxB1-Cre targeted cells (D). However, the medulla appears to be completely contained within the HoxB1 defined region (E). Examination at higher magnification of coronal sections stained for TH in red, LacZ in green, and DAPI-stained nuclei in blue reveals that multiple noradrenergic neuron populations are also targeted by HoxB1, including $\mathrm{A} 5(\boldsymbol{F}), \mathrm{A2}(\boldsymbol{H})$, and $\mathrm{A} 1(\boldsymbol{I})$; however, the $\mathrm{LC}$ located in the dorsal pons is not $(\boldsymbol{G})$. Furthermore, regions critical for regulation of respiratory function are also targeted by HoxB1-Cre, including the RTN $(\boldsymbol{J})$ and the pre-Botzinger complex $(\boldsymbol{K})$. The carotid body, which is responsible for sensing blood oxygen level, is also targeted by HoxB1-Cre, as evident by LacZ staining in reporter animals possessing HoxB1-Cre $(\boldsymbol{L})$ and no staining in the carotid body of reporter animals lacking the HoxB1-Cre $(\boldsymbol{M})$.

In addition, we performed a large battery of behavior tests on the $\mathrm{CKO}$ animals. The CKO animals did not show any abnormalities in the open-field analysis (Fig. 2E), and in a test of social interaction (the partition test), the CKO animals showed no difference compared with the FLOX animals (Samaco et al., 2008) (Fig. $2 F$ ). No difference was observed in the light-dark task, a test of anxiety (Fig. $2 G$ ). In a test of learning and memory, the conditioned fear task, the CKO animals showed the same degree of learning as the FLOX allele (Samaco et al., 2008) (Fig. 2H). Therefore, although MeCP2 function is required within the HoxB1 domain for motor coordination, it is not required within this region for general activity, social function, anxiety, or fearbased learning.

\section{Animals lacking MeCP2 in the HoxB1 domain have normal cortical EEG patterns}

Because seizures are common in RTT (Glaze et al., 2010), we evaluated these mice for cortical electrical abnormalities. NULL animals also show abnormal cortical EEG tracings, with a variety of features that indicate increased excitability. Figure $2 I$ shows a representative epoch from one animal with abnormal synchronous cortical discharge activity. At other times, brief spike bursts and a recorded seizure characterized by an abrupt onset of generalized spike discharges that gradually increased in frequency until the termination of the ictal event were recorded. In contrast, the EEG pattern of the CKO animals looked remarkably normal (representative trace in Fig. $2 J$ ), and no overt seizures were ever observed. These data indicate that the abnormal cortical electrical activity observed in the NULL animals is due to loss of MeCP2 function in the non-HoxB1 domain, likely within the cortex itself.

Removing MeCP2 from the HoxB1 domain disrupts a subset of physiological processes

Because genetic strain effects can dramatically alter phenotypes produced when $\mathrm{MeCP} 2$ function is disrupted, we performed detailed characterization of Mecp $2^{\text {tm1.1Bird/Y }}$ animals (hereafter referred to as "NULL") (Guy et al., 2001) in the same genetic strain 

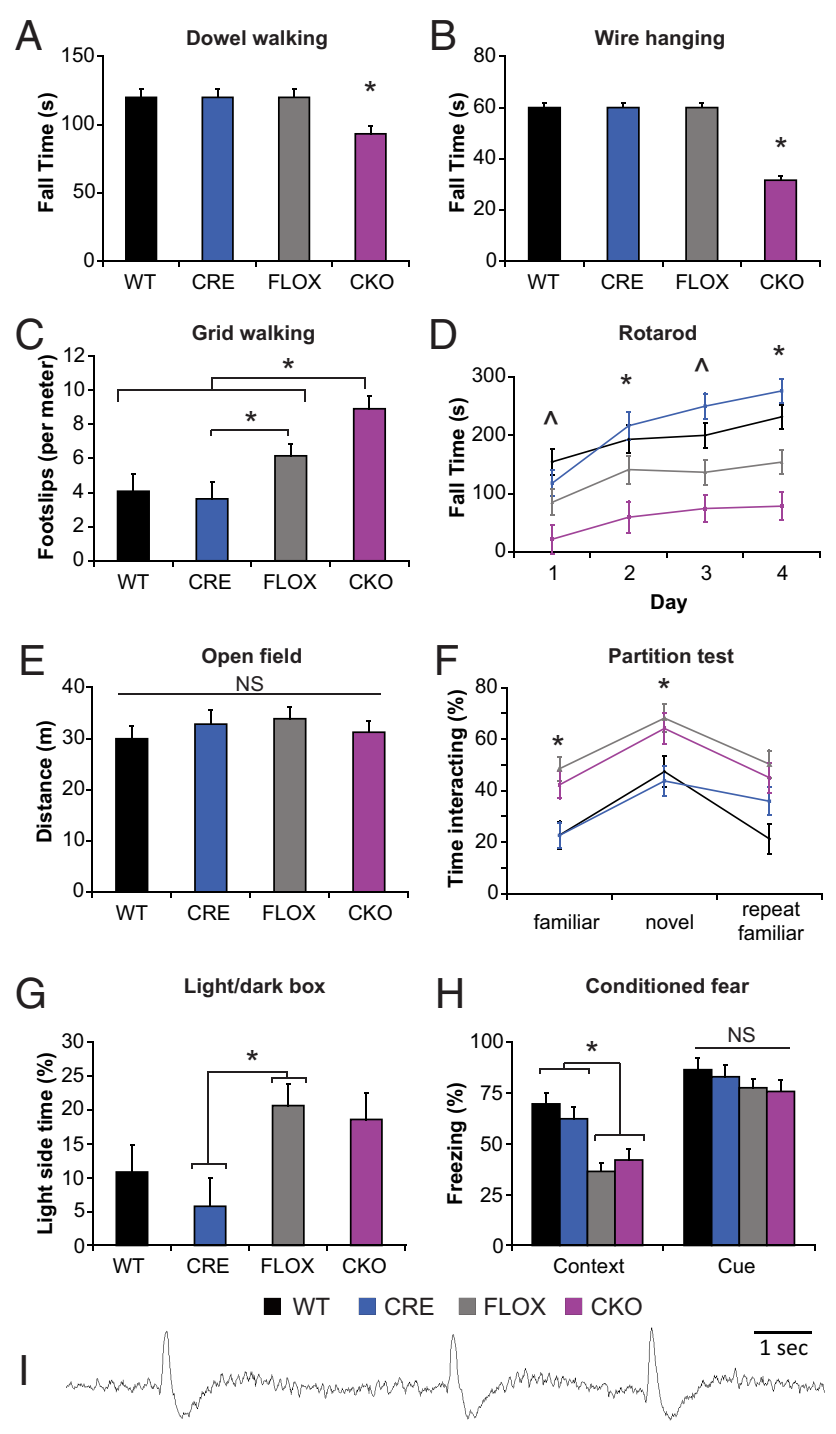

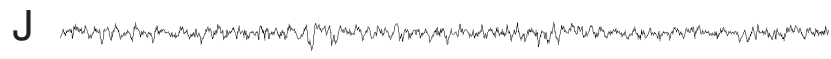

Figure 2. Animals lacking MeCP2 in the hindbrain had motor coordination problems but normal forebrain-associated behaviors. Hindbrain CKO animals were unable to walk on a dowel rod as long as control genotypes ( $A, n=5$ for each genotype) and fell sooner when suspended by their forepaws to a wire ( $B, n=4$ for each genotype). During a 5 min period walking on a wire mesh, CKO animals $(n=10)$ had more footslips per meter traveled than all other genotypes (C). In this assay, the FLOX animals $(n=9)$ also had more footslips than WT $(n=5)$ or CRE animals $(n=5)$, but the CKO had an increased rate of footslips compared with FLOX. CKO ( $n=$ 4) and FLOX $(n=5)$ animals also performed poorly on an accelerating rotating rod compared with WT $(n=5)$ and CRE $(n=5)$ animals (D). On days 2 and 4 , the CKO animals were significantly worse than the FLOX. CKO animals traveled the same distance as control genotypes in the open-field chamber ( $\boldsymbol{E}$, WT $n=9$, CRE $n=9$, FLOX $n=10$, CKO $n=12$ ). In a test of social interaction, the partition task, CKO animals behaved in the same fashion as the FLOX animals, spending more time at the partition when exposed to familiar or novel mice $(\boldsymbol{F}$, WT $n=9$, CRE $n=10, \mathrm{FLOX} n=11, \mathrm{CKO} n=9$ ). Although FLOX animals spend more time in the light side of the light/dark box compared to CRE, the CKO animals were not significantly different from any other genotype ( $G$, WT $n=9$, CRE $n=8$, FLOX $n=14$, CKO $n=9)$. In the conditioned fear task, the $C K O$ and the $F L O X$ animals showed a learning deficit in the context but not the cue portion of the task ( $\boldsymbol{H}, \mathrm{WT} n=8$, CRE $n=7$, FLOX $n=13$, CKO $n=8)$; however, there is no difference between the CKO and the FLOX. Loss of MeCP2 function in the forebrain as seen in NULL animals caused abnormal cortical electrical activity as shown by representative electroencephalogram tracings $(I)$ showing frequent high-amplitude electrical spiking. In contrast, hindbrain CKO animals showed normal cortical electrical activity $(\boldsymbol{J})$ and never displayed the spiking activity seen in the NULL animals. For $\boldsymbol{A}-\boldsymbol{D},{ }^{*} p<0.05$, one-way ANOVA with genotype as factor, background as the CKO animals. In this background, the NULL animals are markedly obese (Fig. $3 A$ ). In contrast, the CKO animals are not overweight and, in fact, weigh less than FLOX littermate control animals from 9 to 10 weeks of life (Fig. 3B). NULL mice show an abnormal response to injected glucose with increased serum glucose at all time points after injection (Fig. 3C). In contrast, the $\mathrm{CKO}$ show normal responses to this glucose challenge (Fig. 3D).

To determine whether the animal models of RTT have cardiac rhythm abnormalities, we characterized the NULL animals with a noninvasive measure of heart rate. NULL animals have decreased basal heart rate (Fig. $3 E$ ), and this finding is reproduced in CKO animals (Fig. $3 F$ ). To further characterize autonomic dysfunction, we measured body temperature with an implanted temperature probe. NULL animals show a decreased basal temperature (Fig. $3 G$ ), which is not present in the CKO animals (Fig. $3 H$ ). Thus, MeCP2 function is required within the hindbrain for normal heart rate, but is dispensable in this region for body temperature. We have previously found that $\mathrm{MeCP} 2$ is required within the hypothalamus for normal weight regulation. Because the hypothalamus is rostral to the boundary of the HoxB1 domain targeted in CKO animals (Fig. 1C), the lack of obesity in CKO animals was expected; however, their underweight phenotype implies an additional role of MeCP2 in the HoxB1 domain for weight regulation. The normal temperature regulation found in these $\mathrm{CKO}$ animals suggests that $\mathrm{MeCP} 2$ function is required in non-HoxB1 lineages for correct temperature homeostasis. Blood oxygenation, as measured by pulse oximeter, was unaffected both in NULL animals (Fig. 3I) and in CKO mice (Fig. 3J).

Loss of MeCP2 from the HoxB1 domain caused early lethality NULL animals died early, with a median survival of $65 \mathrm{~d}$ (Fig. $4 A$ ). Animals that lack MeCP2 in the HoxB1 region showed similar early lethality, with a median survival of $93 \mathrm{~d}$ (Fig. $4 \mathrm{~A}$ ). Additionally, we found similar early death when we removed MeCP2 from the HoxB1 domain using a different "floxed" allele of Mecp2, Mecp2 $2^{\text {tmlJae }}$ (median age of death $=126 \mathrm{~d}, n=13$ ) (Chen et al., 2001; Guy et al., 2001; Pratte et al., 2011). We noted that NULL animals had a progressive decline in their heart rate as they approached death (Fig. $4 B$ ) and a decline in their basal temperature on the day of death (Fig. $4 C$ ), with no evidence of infection as assessed via blood cultures. Additionally, continuous EEG recorded from a subset of moribund NULL animals did not show increased epileptiform or seizure activity. Finally, telemetric recording of cardiac electrical activity in moribund animals showed progressive bradycardia, and in a few cases asystole, but no evidence of other cardiac rhythm abnormalities that could cause death. We noted a similar decline in heart rate (Fig. $4 B$ ) and body temperature (Fig. 4C) in the $\mathrm{CKO}$ animals on the day of death. Therefore, removing MeCP2 from the hindbrain caused death similar to that observed in NULL animals, indicating that MeCP2 is necessary within the HoxB1 domain for normal lifespan.

$\leftarrow$

$p<0.05$ for Tukey post hoc pairwise comparisons between CKO and all other genotypes. $\wedge p<$ 0.05 , one-way ANOVA with genotype as factor, $p<0.05$ for Tukey post hoc pairwise comparisons between CKO and CRE or WT. $\boldsymbol{F}^{*} p<0.05$, one-way ANOVA with genotype as factor, $p<0.05$ for Tukey post hoc pairwise comparisons between FLOX or CKO and WT or CRE. G, $\boldsymbol{H}^{*} p<0.05$, one-way ANOVA with genotype as factor, $p<0.05$ for Tukey post hoc pairwise comparisons as indicated. NS, Not significant. Error bars indicate SEM. 

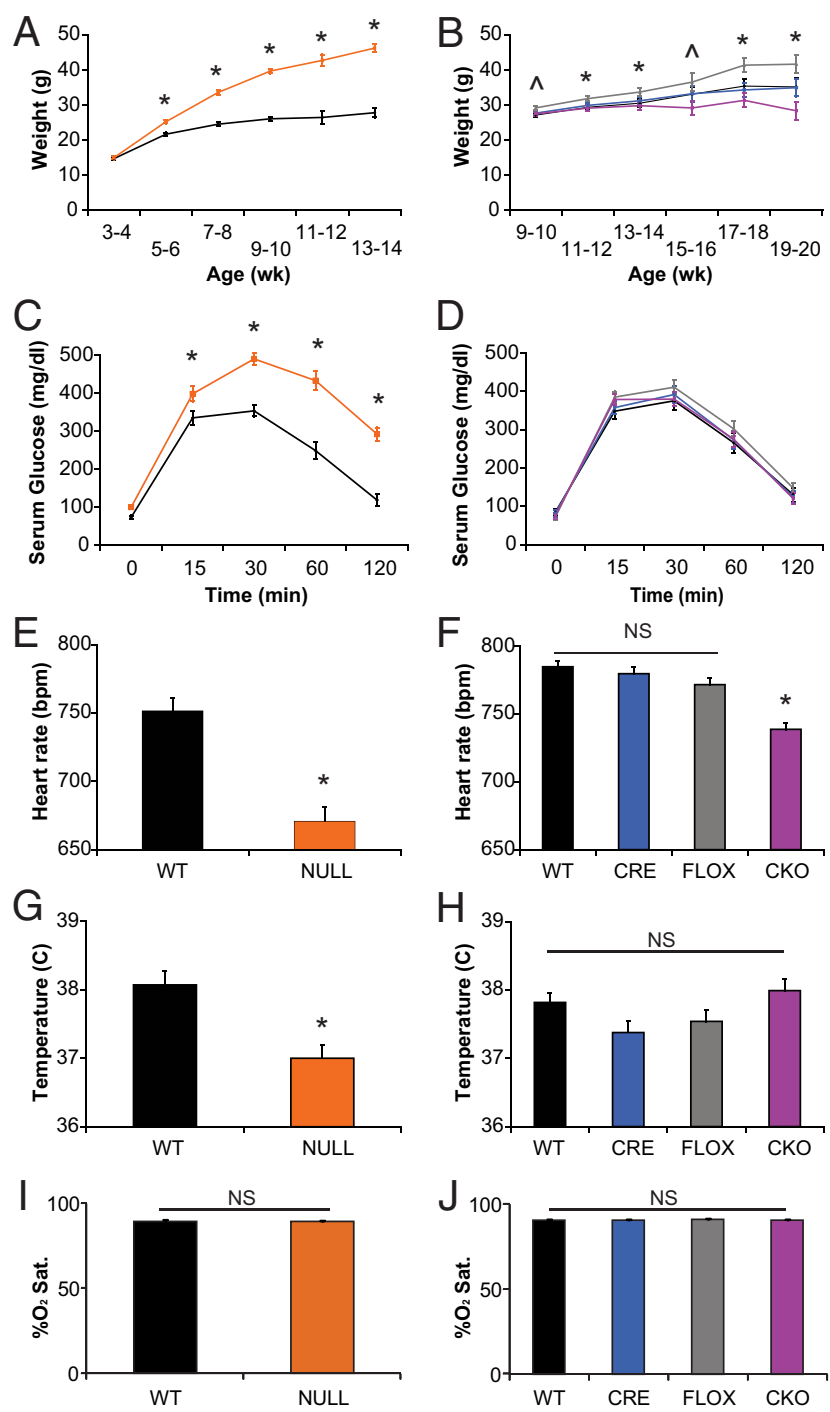

$\square$ WT $\square$ NULL

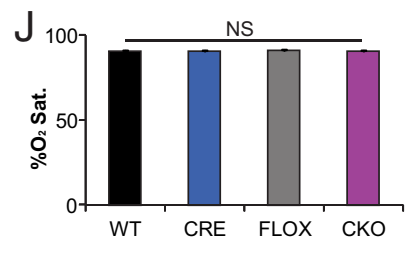

-WT $\square$ CRE $\square$ FLOX $\square$ CKO

Figure 3. Abnormalities to physiological systems when MeCP2 function was disrupted in the hindbrain. NULL animals were markedly obese from 5 to 6 weeks of life $(\boldsymbol{A}$, WT, black, $n>24$ for each time point; NULL, orange, $n>21$ for each time point). Hindbrain (KO animals did not show this increased weight phenotype (B); in fact, CKO animals (purple, $n>4$ for each time point) weighed less than FLOX littermates (gray, $n>4$ for each time point) from 9 weeks of life, and ultimately weigh less than all the control genotypes (WT, black, $n>4$; CRE, blue, $n>4$ ) after 17 weeks of life. As expected for obese animals, the NULL animals had an abnormal response to injected glucose, showing a dramatic increase in blood glucose $15 \mathrm{~min}$ after injection (C, WT $n=7$, NULL $n=4)$. In contrast, hindbrain CKO animals did not show abnormally increased blood glucose after injection of glucose ( $\boldsymbol{D}$, WT $n=4$, CRE $n=2$, FLOX $n=9$, CKO $n=5)$. NULL animals $(n=9)$ showed a decreased heart rate compared to wild-type littermate controls $(\boldsymbol{E}, \mathrm{WT}, n=8)$. Similarly, hindbrain CKO animals $(n=10)$ had a decreased heart rate compared pairwise with all the control genotypes $(\boldsymbol{F}$, WT $n=15$; CRE $n=10$, FLOX $n=9)$. Body temperature was reduced in NULL animals $(n=8)$ compared with WT $(\boldsymbol{G}, n=8)$; in contrast, body temperature was not reduced in the CKO animals $(\boldsymbol{H}, \mathrm{WT} n=14$, CRE $n=10$, FLOX $n=9,(K 0 n=10)$. Blood oxygen saturation as measured at the tail via pulse oximeter indicated no statistical difference between WT and NULL animals (I, WT $n=8, \mathrm{NULL}, n=9)$, nor was there a measurable difference between CK0 animals and littermate controls (J, WT $n=$ 15; CRE $n=10$, FLOX $n=9$, CKO $n=10$ ). For panels comparing NULL to WT, ${ }^{*} p<0.05$, one-way ANOVA $(A, C, E, G)$. For panels comparing CKO to the three other possible genotypes (WT, CRE, FLOX), ${ }^{*}$ means that there is an overall difference between the genotypes assessed using one-way ANOVA with genotype as a factor, and that there is a $p<0.05$ post hoc pairwise difference between CKO and all other genotypes assessed using Tukey HSD post hoc testing ( $\boldsymbol{B}$, D). ${ }^{\wedge}$ post hoc $p<0.05$ for pairwise comparison of FLOX to CKO only. NS, Not significant. Error bars indicate SEM.
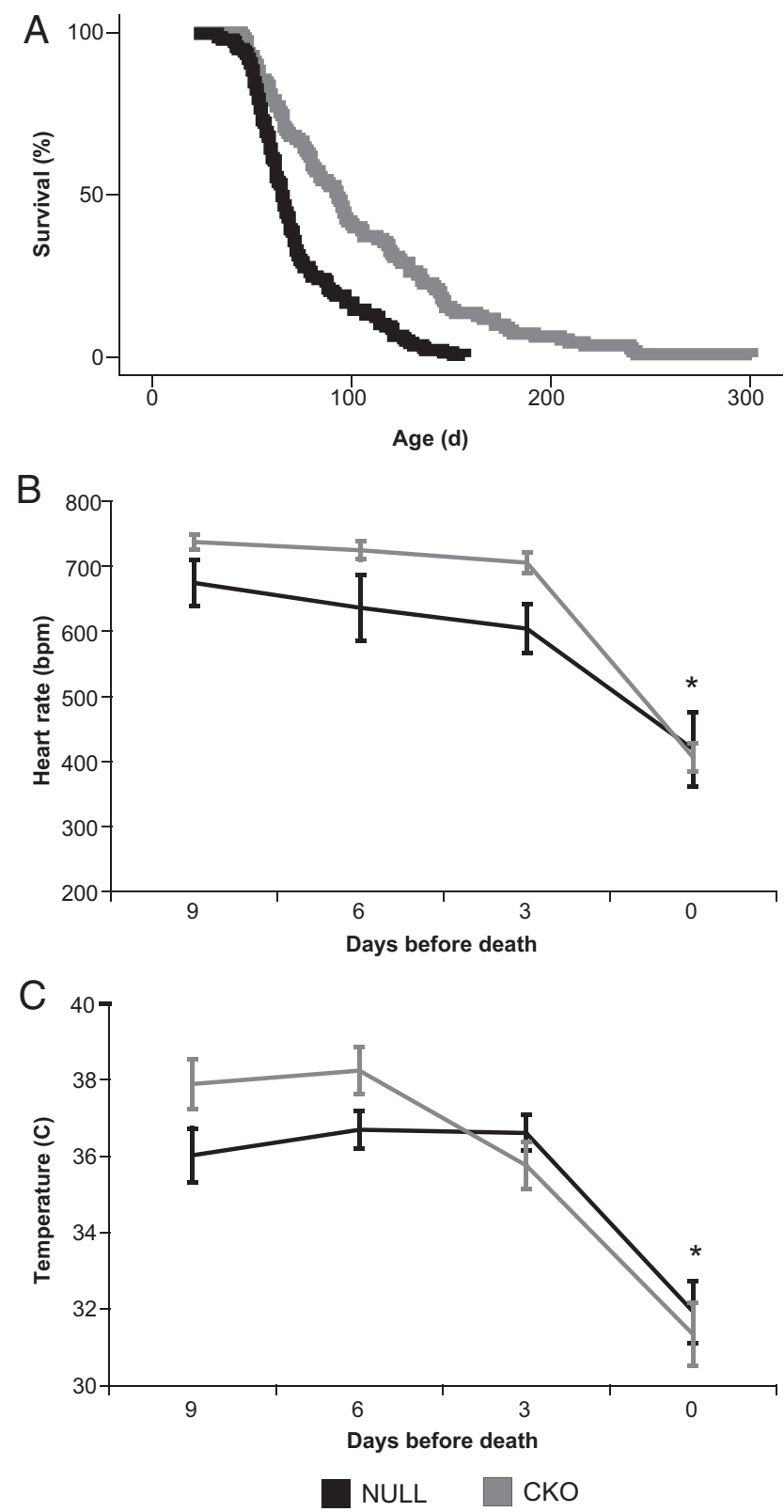

Figure 4. Loss of MeCP2 in the hindbrain leads to early lethality. NULL mice ( $n=137$, black) showed early lethality with a median survival of $65 \mathrm{~d}(\boldsymbol{A})$. Hindbrain CKO mice (CKO, gray, $n=$ $110)$ had early lethality with a mean survival of $93 d(\boldsymbol{A})$. As NULL animals progressed toward death, their heart rates progressively declined ( $\boldsymbol{B}$, black line, $n>3$ for each time point), and on the day of death, their body temperature dropped dramatically ( $\boldsymbol{C}$, black line). In a similar fashion, hindbrain CKO animals showed a decline in both heart rate ( $\boldsymbol{B}$, gray line, $n>5$ for each time point) and temperature (C, gray line) on the day of death. $\boldsymbol{B}, \boldsymbol{C},{ }^{*} p<0.05$ pairwise difference between day 0 and all other days; error bars indicate SEM.

\section{Animals lacking MeCP2 in the HoxB1 domain exhibited} abnormal breathing responses

Abnormal patterns of breathing are present in children with MECP2 mutations and have been reported in NULL mice (Viemari et al., 2005; Ogier et al., 2007; Voituron et al., 2009). To accurately compare our CKO animals to NULL animals, we performed detailed characterizations in the same genetic strain. In this strain, the NULL animals had an increased mean breathing rate compared with WT controls (Fig. 5A). This increased breathing rate was not accompanied by an alteration in tidal volume unless normalized for body weight (Fig. $5 B, C$ ); however, the 

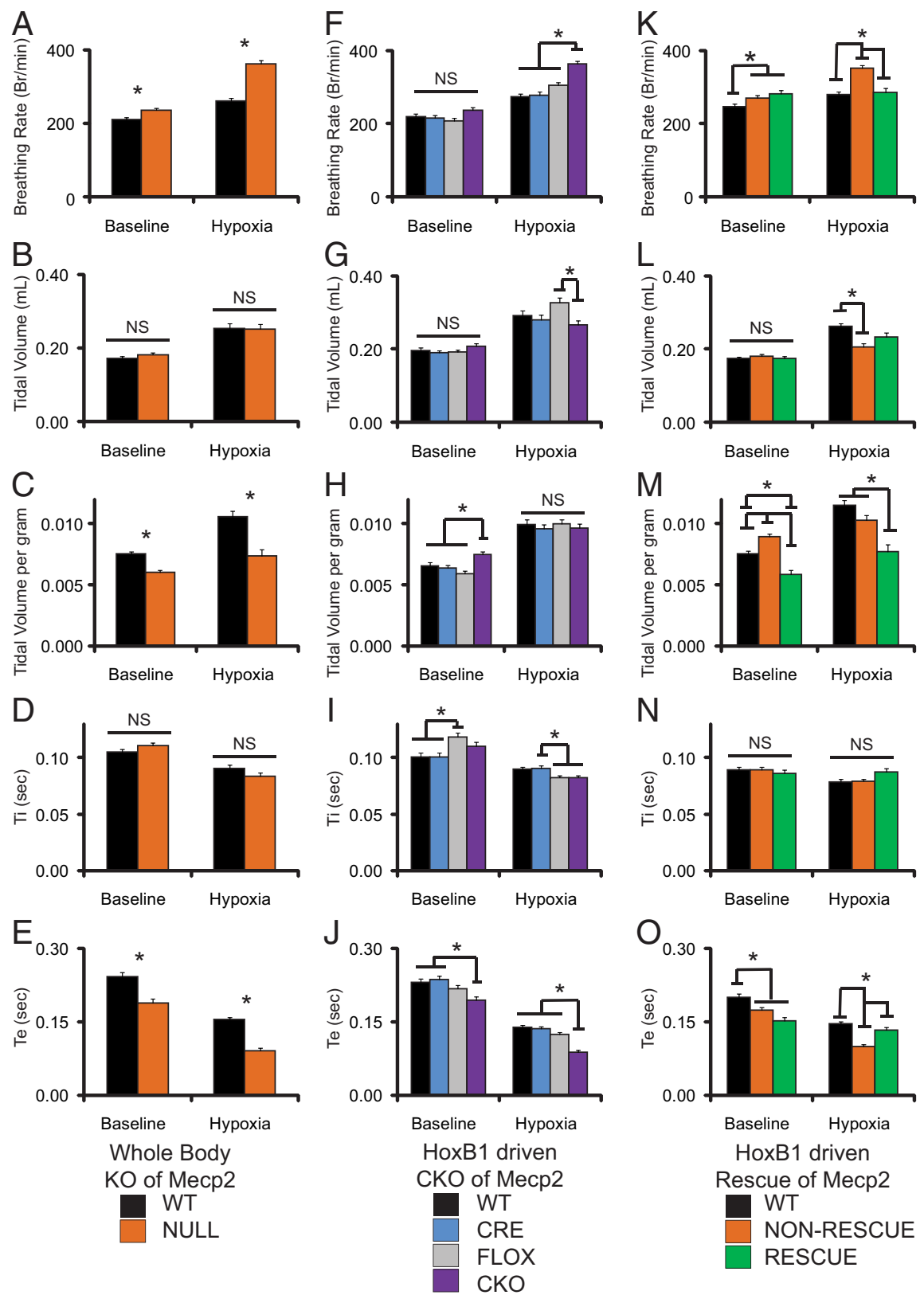

$\mathrm{N}$
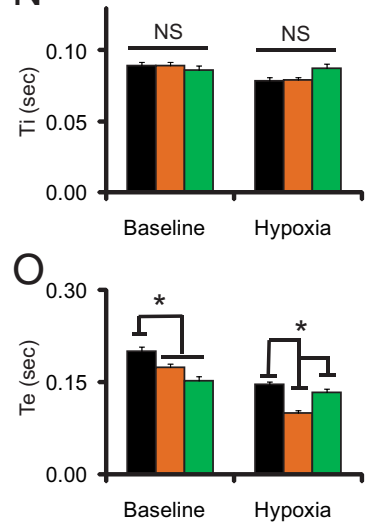

HoxB1 driven

Rescue of Mecp2

WT

NON-RESCUE RESCUE

Figure 5. Abnormal baseline and hypoxia induced breathing in mice with disrupted MeCP2 function. Breathing parameters during baseline conditions (Baseline) and hypoxic challenge with $10 \% 0_{2}$ balance $\mathrm{N}_{2}$ (Hypoxia) were investigated by unrestrained plethysmography in mice with disrupted MeCP2. Removal of MeCP2 function from the whole body, as demonstrated by NULL animals ( $n=47$ baseline, $n=10$ hypoxia), causes an increased breathing rate compared to WT animals ( $n=50$ baseline, $n=$ 13 hypoxia) during baseline as well as during hypoxic challenge $(\boldsymbol{A})$. There is no apparent difference in tidal volume $(\boldsymbol{B})$; however, when normalized to body weight the tidal volume of NULL animals is decreased compared to WT during both baseline and hypoxia $(C)$, likely related to the obesity phenotype observed in the animals (see Fig. $3 A$ ). The average inspiratory time is not significantly different between NULL and WT animals $(\boldsymbol{D})$, making a decrease in expiratory time the primary contribution to the increased breathing rate observed in NULL animals during baseline and hypoxia (E). Removal of MeCP2 from the hindbrain with HoxB1-Cre results in CKO animals ( $n=26$ baseline, $n=15$ hypoxia) exhibiting normal baseline breathing frequency compared to control littermates (WT $n=23$ baseline, $n=12$ hypoxia; CRE $n=26$ baseline, $n=13$ hypoxia; FLOX $n=27$ baseline, $n=14$ hypoxia) but reproducing the increased rate during hypoxia seen in NULL animals $(\boldsymbol{F})$. Also similar to NULL animals, CKO animals exhibit a tidal volume that is similar to WT during baseline and hypoxia (G). However, the FLOX animals exhibited an increased tidal volume relative to the CKO during hypoxia. Normalization of tidal volume to body weight results in a significantly increased tidal volume of CKO animals compared to control genotypes during baseline, likely due to the slight underweight phenotype of the CKO animals; during hypoxia, there is no significant difference in weight-normalized tidal volume $(\boldsymbol{H})$. The average inspiratory time of the CKO was not significantly different from the control genotypes at baseline, although the $F L O X$ animals showed an increased baseline inspiratory time relative to WT and CRE littermates; during hypoxia, both the CKO and FLOX mice exhibited a slightly decreased inspiratory time relative to CRE littermates (I). Average expiratory time was slightly decreased in CKO relative to WT and CRE littermates during baseline; decreased expiratory time relative to all control genotypes was also the major contributor to the increased breathing rate during hypoxia in the CKO animals (J). Restoration of MeCP2 limited to the hindbrain with HoxB1-Cre- obesity phenotype of the NULL animals likely drove this difference in the weight normalized tidal volume. Examination of the average inspiratory and expiratory times revealed that a decrease in the expiratory time drove the increased baseline breathing rate observed in NULL animals (Fig. 5D,E). In contrast, the hindbrain CKO animals did not recapitulate this increased baseline breathing rate (Fig. $5 F$ ). Similarly, the CKO did not exhibit an altered tidal volume unless normalized for body weight (Fig. 5G,H); however, the underweight phenotype of the $\mathrm{CKO}$ animals likely contributed to this phenotype. The CKO exhibited a baseline inspiratory time between that of the FLOX animals and the WT and CRE animals, although not significantly different from either (Fig. 5I). Also, although the expiratory time of the CKO animals was significantly decreased relative to WT and CRE animals, it was not different from FLOX littermates and not sufficient to recapitulate the increased baseline breathing rate observed in the NULL animals (Fig. $5 J$ ).

NULL animals have also been reported to show abnormal breathing responses to challenges with decreased oxygen concentrations $\left(10 \% \mathrm{O}_{2}\right.$, balance $\mathrm{N}_{2}$ - hypoxia) (Roux et al., 2008; Voituron et al., 2009). We found that NULL animals showed an increased respiratory rate relative to WT littermates in response to hypoxia (Fig. $5 A$ ), indicative of a deficit in the hypoxic ventilatory decline associated with exposure to acute hypoxia (Teppema and Dahan, 2010). The tidal volume of NULL

\section{$\leftarrow$}

driven expression of a conditional rescue allele of $M e c p 2$ in RESCUE animals ( $n=17$ baseline, $n=7$ hypoxia) results in a failure to rescue to the increased baseline breathing rate observed in both NULL and NON-RESCUE animals ( $n=29$ baseline, $n=15$ hypoxia) relative to WT ( $n=29$ baseline, $n=14$ hypoxia); however, the breathing rate of RESCUE animals during hypoxia is restored to WT levels with NON-RESCUE animals demonstrating an increased breathing rate similar to NULL animals ( $\boldsymbol{K}$ ). At baseline, there is no significant difference in tidal volume between RESCUE animals and WT littermates; however, the NON-RESCUE animals have a decreased tidal volume during hypoxia relative to WT littermates $(\boldsymbol{L})$. When normalized to body weight, RESCUE animals exhibit a decreased tidal volume during baseline and hypoxia $(\boldsymbol{M})$, likely due to their overweight phenotype (see Fig. 7A). Average inspiratory time is not significantly different between RESCUE animals and control genotypes during baseline or hypoxia $(\boldsymbol{N})$. However, similar to NULL and CKO animals the major contribution to the increased breathing rate in NON-RESCUE animals is from a decrease in expiratory time, which is shared by RESCUE animals during baseline conditions but restored to WT levels during hypoxia (0). ${ }^{*} p<0.05$, one-way ANOVA with genotype as factor, $p<0.05$ for Tukey post hoc pairwise comparisons as indicated. NS, Not significant. Error bars indicate SEM. 

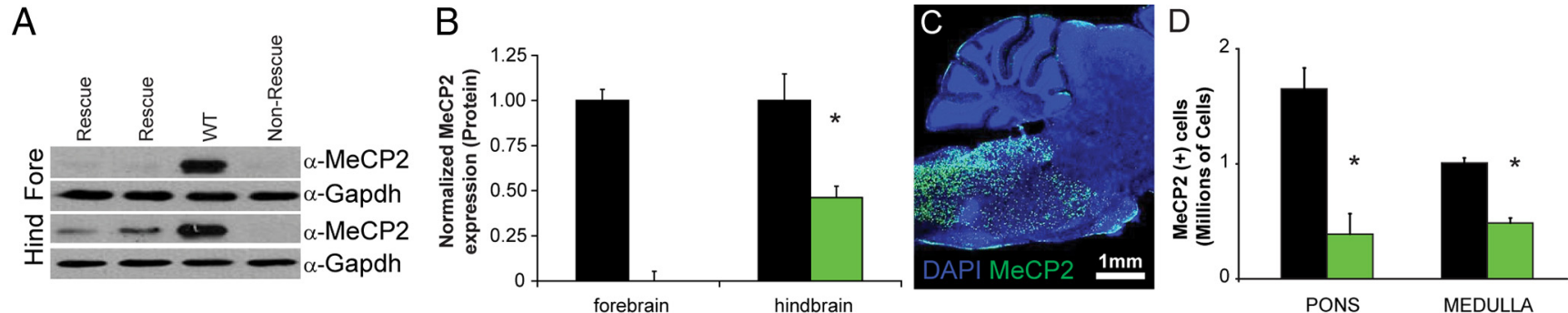

PONS (DAPI MeCP2)
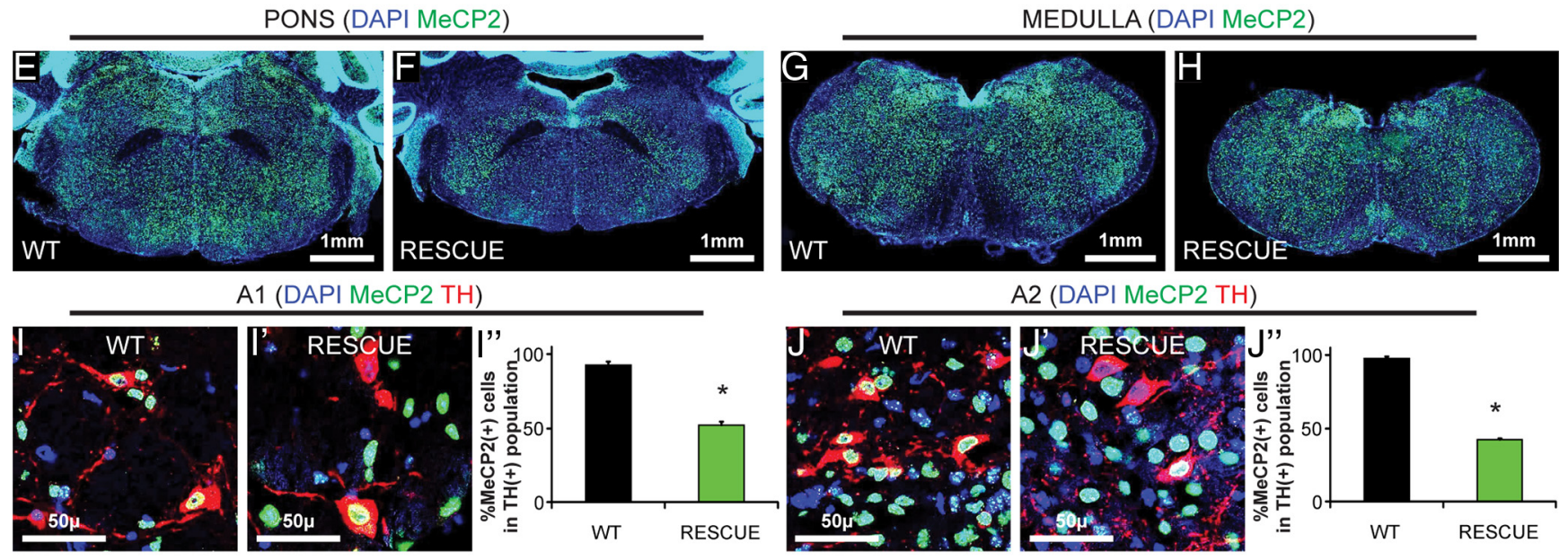

A5 (DAPI MeCP2 TH)

LC (DAPI MeCP2 TH)
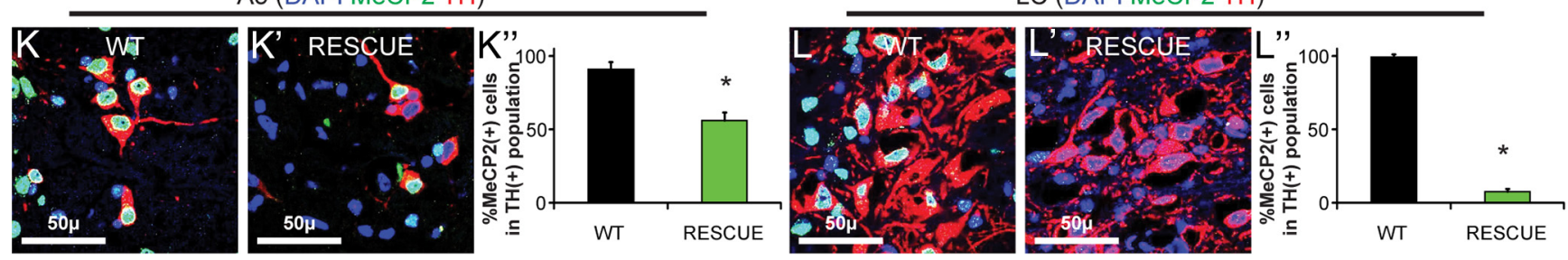

NTS (DAPI MeCP2 SST)

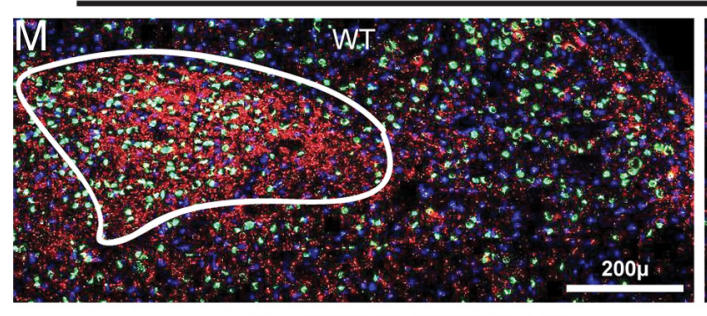

RTN (DAPI MeCP2 VAchT)

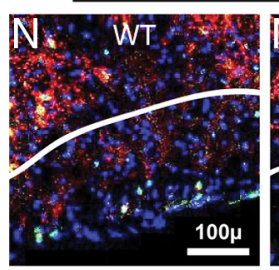

SCG (DAPI MeCP2 TH)
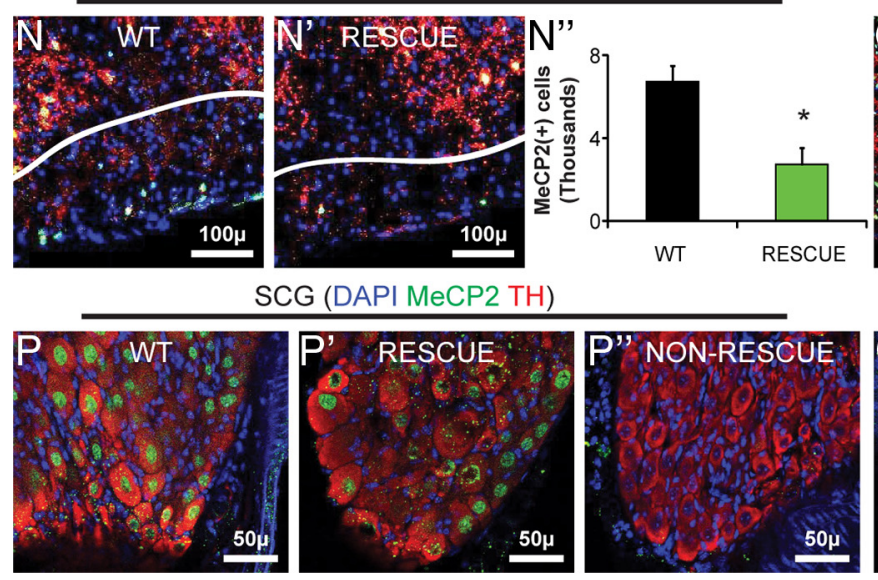
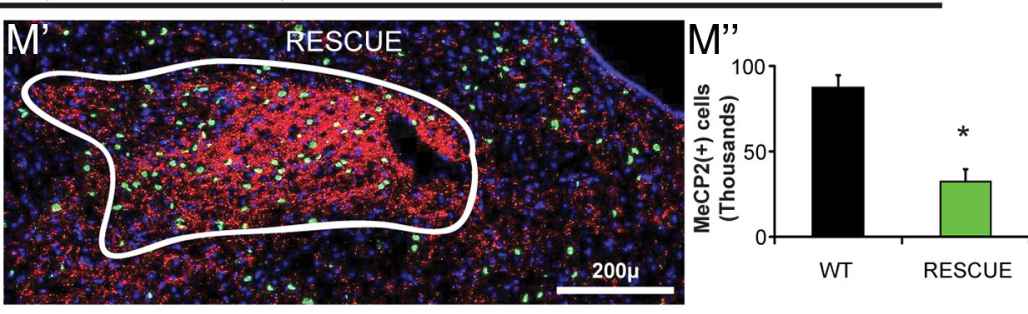

Pre-Botz (DAPI MeCP2 NK1R)
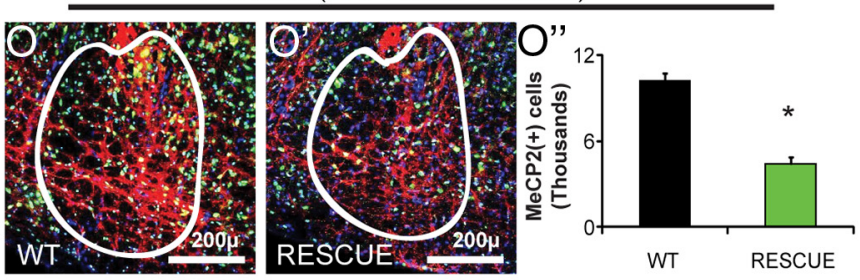

Carotid Body (DAPI MeCP2 TH)
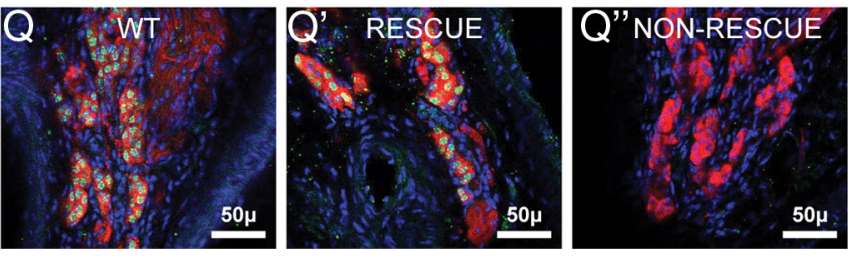

Figure 6. Spatially restricted induction of MeCP2 in the hindbrain. MeCP2 protein expression was induced in the hindbrain (Hind) of RESCUE animals; however, no MeCP2 is expressed in the forebrain (Fore) of these animals $(\boldsymbol{A})$. In comparison, there are equal MeCP2 levels in the forebrain and hindbrain of control WT animals, and a lack of (Figure legend continues.) 
animals during hypoxia was similar to WT littermates; however, the obesity phenotype of the NULL animals contributes to a decreased weight normalized tidal volume during hypoxia (Fig. $5 B, C)$. Assessment of the average inspiratory and expiratory times revealed that a decrease in the expiratory time also drove the increased hypoxic breathing rate observed in NULL animals (Fig. $5 D, E$ ). Removing MeCP2 from the HoxB1 domain recapitulated this increased hypoxic breathing rate relative to control littermates (Fig. 5F). The tidal volume of CKO animals was not significantly different from WT littermates, although FLOX animals did exhibit an increased tidal volume versus the CKO (Fig. $5 G)$. Furthermore, there were no significant differences between $\mathrm{CKO}$ and control littermates for tidal volume normalized to bodyweight during hypoxia. The inspiratory time of FLOX and CKO animals was slightly decreased versus CRE littermates (Fig. $5 I$ ); however, the decreased expiratory time of the CKO animals versus control littermates during hypoxia provided a larger contribution to the increased breathing rate during hypoxia (Fig. $5 J$ ).

Together these data suggest that the increased respiratory rates resulting from $\mathrm{MeCP} 2$ dysfunction during baseline conditions or challenges with hypoxia possess different origins. Baseline abnormalities likely originate from structures rostral to the HoxB1 domain and abnormalities during hypoxia from structures within the HoxB1 domain.

\section{Restoring MeCP2 function within the HoxB1 domain rescued autonomic abnormalities and lifespan}

To determine whether MeCP2 expression in this tissue is sufficient to prevent early death and autonomic abnormalities, we restored $\mathrm{MeCP} 2$ exclusively within the HoxB1 domain using a recently engineered stop-flox or "RESCUE" allele of Mecp2, Mecp $2^{\text {tm2Bird }}$ (Guy et al., 2007). In animals lacking Crerecombinase, no Mecp2 mRNA or MeCP2 protein is expressed (NON-RESCUE), and thus these animals are indistinguishable from NULL mice. MeCP2 expression can be induced with exposure to Cre, such as the HoxB1 $1^{\text {cre }}$ (RESCUE). Western blotting showed that MeCP2 expression was absent from both the fore-

\footnotetext{
(Figure legend continued.) observable MeCP2 protein in either the hindbrain or forebrain of NON-RESCUE animals where MeCP2 expression had not been induced. Quantification by densitometry $(\boldsymbol{B})$ revealed that the level of MeCP2 induced in the hindbrains of RESCUE animals $(n=$ 7) was $46 \%$ of the MeCP2 in the hindbrains of WT animals $(n=5)$. In contrast, MeCP2 protein was negligible within the forebrain of the RESCUE animals. A sagittal section through the hindbrain of a RESCUE animal shows MeCP2 expression in the brainstem with a rostral boundary in the pons $(C)$ expected from the HoxB1-Cre (compare to Fig. 1C). Stereological quantification of the number of MeCP2-expressing cells ( $\boldsymbol{D})$ indicate that RESCUE animals $(n=3)$ exhibit $\sim 25 \%$ of the number of $\mathrm{MeCP} 2(+)$ cells in the pons and $50 \%$ of the number of $\mathrm{MeCP} 2(+)$ cells in the medulla observed in WT animals $(n=3)$. $\boldsymbol{E}-\boldsymbol{H}$ illustrate coronal sections taken through the pons of WT $(\boldsymbol{E})$ and RESCUE $(\boldsymbol{F})$ as well as the medulla of WT $(\boldsymbol{G})$ and RESCUE $(\boldsymbol{H})$ animals. Examination of the efficiency of rescue within the noradrenergic neurons throughout the hindbrain $\left(I-L^{\prime \prime}\right.$, WT $n=3$, RESCUE $\left.n=3\right)$ indicate that RESCUE animals express MeCP2 in $\sim 50 \%$ of the TH-expressing cells of the A1 $\left(\boldsymbol{I}-\boldsymbol{I}^{\prime \prime}\right), \mathrm{A} 2\left(\boldsymbol{J}-\boldsymbol{J}^{\prime \prime}\right)$, and A5 $\left(\boldsymbol{K}-\boldsymbol{K}^{\prime \prime}\right)$ populations, which are targeted by HoxB1-Cre, while the LC is mostly not targeted by HoxB1-Cre, with $<10 \%$ of TH-expressing neurons expressing MeCP2 in RESCUE animals $\left(L-L^{\prime \prime}\right)$; nearly $100 \%$ of THexpressing cells also expressed MeCP2 in WT animals across the quantified noradrenergic populations. The efficiency of rescue was also determined by stereological quantification of MeCP2expressing cells in WT $(n=3)$ and RESCUE $(n=3)$ animals within the NTS $\left(M-M^{\prime \prime}\right)$, the RTN $\left(\boldsymbol{N}-\boldsymbol{N}^{\prime \prime}\right)$, and the pre-Botzinger complex $\left(\mathbf{O}-\mathbf{O}^{\prime \prime}\right)$; across these regions, RESCUE animals possessed $\sim 40 \%$ of the MeCP2-expressing cells observed in WT animals. MeCP2 was also expressed in the superior cervical ganglion of WT $(\boldsymbol{P})$ and RESCUE $\left(\boldsymbol{P}^{\prime}\right)$ animals, but not NONRESCUE animals $\left(\boldsymbol{P}^{\prime \prime}\right)$. Similarly, the carotid body also showed MeCP2 expression in WT (Q) and RESCUE $\left(\boldsymbol{Q}^{\prime}\right)$ mice, but not NON-RESCUE animals $\left(\boldsymbol{Q}^{\prime \prime}\right){ }^{*} p<0.05$, one-way ANOVA with genotype as factor. Error bars indicate SEM.
}

brain and the hindbrain of NON-RESCUE animals, whereas RESCUE animals showed MeCP2 expression within the hindbrain but not the forebrain (Fig. 6A). The amount of MeCP2 protein induced within the hindbrain was $<50 \%$ of that seen in WT control animals (Fig. 6B). Stereological quantification of MeCP2-expressing cells throughout the pons and medulla revealed that RESCUE animals possess fewer MeCP2-expressing cells in the hindbrain than WT littermates (WT, $n=3$, pons $1.65 \times 10^{6} \pm 0.44 \times 10^{6}$, medulla $1.01 \times 10^{6} \pm 0.08 \times 10^{6}$; RESCUE, $n=3$, pons $0.39 \times 10^{6} \pm 0.07 \times 10^{6}$, medulla $0.49 \times$ $10^{6} \pm 0.08 \times 10^{6}$; AVG $\pm \mathrm{SD}$ ) (Fig. $\left.6 \mathrm{D}\right)$.

RESCUE animals possessed regional restriction of MeCP2expressing cells expected from the HoxB1 ${ }^{\text {cre }}$ (Fig. 6C, E-H- compare to Fig. $1 C-E$ ). To determine whether the efficiency of MeCP2 expression in RESCUE animals reflected a general phenomenon or biased restoration to specific populations of neurons, we examined the efficiency of MeCP2 expression in several regions implicated in respiratory and autonomic control. We quantified the number of noradrenergic neurons and the fraction that expressed MeCP2 in WT and RESCUE animals (Fig. 6I-L"). WT animals expressed detectable MeCP2 in nearly $100 \%$ of the noradrenergic neurons quantified [WT; A1: $1011 \pm 101 \mathrm{TH}(+)$ cells, $92.5 \pm 6.4 \% \mathrm{TH}(+) \mathrm{MeCP} 2(+)$; A2: $1435 \pm 264 \mathrm{TH}(+)$ cells, $97.8 \pm 0.5 \% \mathrm{TH}(+) \mathrm{MeCP} 2(+)$; A5: $477 \pm 76 \mathrm{TH}(+)$ cells, $90.7 \pm 13.7 \% \mathrm{TH}(+) \mathrm{MeCP} 2(+) ; \mathrm{LC}: 3043 \pm 434 \mathrm{TH}(+)$ cells, $99.1 \pm 0.8 \% \mathrm{TH}(+) \mathrm{MeCP} 2(+) ; n=3$; $\mathrm{AVG} \pm \mathrm{SD}]$; rescue animals expressed detectable MeCP2 in approximately half of the noradrenergic neurons of the $\mathrm{A} 1, \mathrm{~A} 2$, and $\mathrm{A} 5$ populations and practically none of the LC neurons, which lie outside of the HoxB1 domain [RESCUE; A1: $1332 \pm 154 \mathrm{TH}(+)$ cells, $52.0 \pm 3.8 \%$ $\mathrm{TH}(+) \mathrm{MeCP} 2(+) ; \mathrm{A} 2: 1844 \pm 237 \mathrm{TH}(+)$ cells, $42.1 \pm$ 4.2\% TH(+)MeCP2(+); A5: $760 \pm 513 \mathrm{TH}(+)$ cells, $56.1 \pm$ $10.2 \% \mathrm{TH}(+) \mathrm{MeCP} 2(+)$; LC: $2475 \pm 538 \mathrm{TH}(+)$ cells, $7.3 \pm 7.1 \% \mathrm{TH}(+) \mathrm{MeCP} 2(+) ; n=3 ; \mathrm{AVG} \pm \mathrm{SD}]$; and NONRESCUE animals expressed detectable MeCP2 in none of the noradrenergic populations tested [NON-RESCUE; A1: $1533 \pm 300$ $\mathrm{TH}(+)$ cells, $0 \pm 0 \% \mathrm{TH}(+) \mathrm{MeCP} 2(+)$; $\mathrm{A} 2: 1883 \pm 159 \mathrm{TH}(+)$ cells, $0 \pm 0 \% \mathrm{TH}(+) \mathrm{MeCP} 2(+)$; A5: $680 \pm 233 \mathrm{TH}(+)$ cells, $0 \pm$ $0 \% \mathrm{TH}(+) \mathrm{MeCP} 2(+)$; LC: $2795 \pm 484 \mathrm{TH}(+)$ cells, $0 \pm 0 \%$ $\mathrm{TH}(+) \mathrm{MeCP} 2(+) ; n=3 ; \mathrm{AVG} \pm \mathrm{SD}]$. To estimate the efficiency of $\mathrm{MeCP} 2$ expression in the NTS, the major relay center for many autonomic and respiratory reflex functions, we quantified the MeCP2-expressing cells contained within somatostatin-stained regions of the dorsal medulla (Fig. $6 M, M^{\prime}, M^{\prime \prime}$ ) [WT 87,476 $\pm 17,332$ MeCP2 $(+)$ cells; RESCUE 32,382 $\pm 6362 \mathrm{MeCP} 2(+)$ cells; NONRESCUE $0 \pm 0 \mathrm{MeCP} 2(+)$ cells; $n=3$ per genotype; $\mathrm{AVG} \pm \mathrm{SD}]$. To estimate the efficiency of MeCP2 expression in the RTN, a major respiratory control center, we quantified MeCP2-expressing cells ventral to the facial nucleus (Fig. $6 N, N^{\prime}, N^{\prime \prime}$ ) [WT $6725 \pm 1815$ MeCP2 $(+)$ cells; RESCUE $2740 \pm 719 \mathrm{MeCP} 2(+)$ cells; NONRESCUE $0 \pm 0 \mathrm{MeCP} 2(+)$ cells; $n=3$ per genotype; $\mathrm{AVG} \pm \mathrm{SD}]$. To estimate the efficiency of MeCP2 expression in the pre-Botzinger complex, the major respiratory rhythm generator, we quantified the MeCP2-expressing cells in the somatostatin and NK1R-expressing region ventral to the nucleus ambiguus (Fig. 6O, $O^{\prime}, O^{\prime \prime}$ ) [WT $10,201 \pm 608 \mathrm{MeCP} 2(+)$ cells; RESCUE $4400 \pm 1092 \mathrm{MeCP} 2(+)$ cells; NON-RESCUE $0 \pm 0 \mathrm{MeCP} 2(+)$ cells; $n=3$ per genotype; $\mathrm{AVG} \pm \mathrm{SD}]$. Furthermore, RESCUE animals also restored MeCP2 expression to peripheral regions, including the superior cervical ganglion (Fig. 6P, $P^{\prime}, P^{\prime \prime}$ ) and the carotid body (Fig. 6Q, $Q^{\prime}, Q^{\prime \prime}$ ).

The NON-RESCUE animals showed early lethality similar to that observed in the NULL animals, with a median survival of $95 \mathrm{~d}$ (Fig. 7A). In contrast, despite our observation that only 

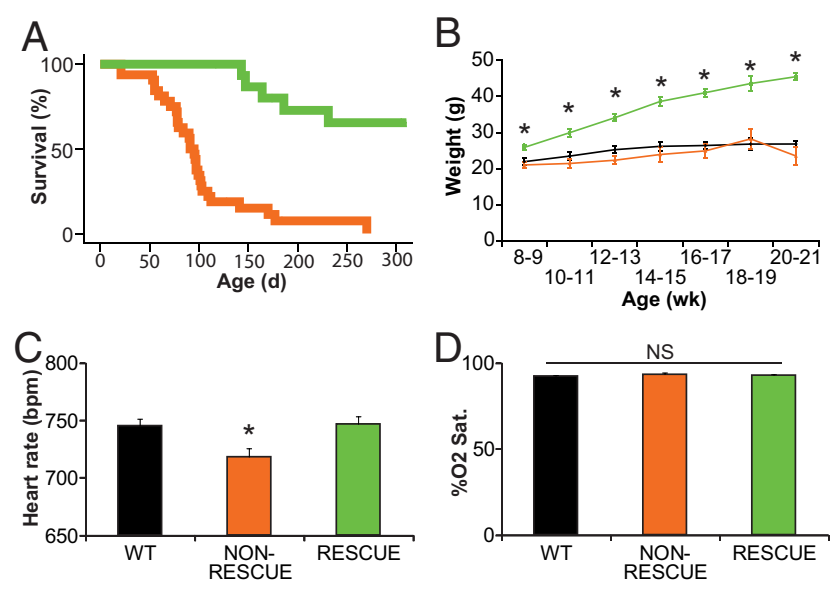

WT NON-RESCUE RESCUE

Figure 7. Inducing expression of $\mathrm{Me} C \mathrm{P} 2$ in the hindbrain rescued early lethality and heart rate abnormality. NON-RESCUE animals showed early lethality ( $\boldsymbol{A}$, orange, $n=32$, median age of death $=104 \mathrm{~d}$ ) similar to that seen in the complete NULL animals (compare with Fig. $4 A$ ). In contrast, RESCUE animals ( $\boldsymbol{A}$, green, $n=16,5$ deaths) showed increased survival, with the majority surviving $>45$ weeks ( $p<0.0001$ comparing NON-RESCUE to RESCUE), at which time some animals were killed to isolate tissue for histological and molecular analysis. Five RESCUE animals lived $>1$ year. The RESCUE animals were overweight compared with the control genotypes $(\boldsymbol{B})$. These RESCUE animals $(n=14)$ had a normal heart rate, indistinguishable from WT $(n=21)$, but markedly elevated compared with the decreased heart rate observed in the NON-RESCUE animals ( $C$, NON-RESCUE $n=13$ ). Blood oxygen saturation as measured at the tail via pulse oximeter indicated no statistically difference between WT, NON-RESCUE, and RESCUE animals ( $\boldsymbol{D}$, WT $n=21$, NON-RESCUE $n=13$, RESCUE $n=14$ ). ${ }^{*} p<0.05$, one-way ANOVA with genotype as factor and $p<0.05$ for Tukey HSD post hoc pairwise comparisons between RESCUE and all other genotypes (B) or NON-RESCUE and all other genotypes (C). NS, Not significant. Error bars indicate SEM.

Table 1. Comparison of phenotypes reproduced in regional knock-out and rescued in regional re-expression of $\mathrm{MeCP} 2$ function

\begin{tabular}{lccc}
\hline Phenotype & NULL & CKO & RESCUE \\
\hline Weight & $\uparrow$ & $\downarrow$ & $\uparrow$ \\
Temperature & $\downarrow$ & Normal & ND \\
Heart rate & $\downarrow$ & $\downarrow$ & Rescued \\
Basal breathing & $\uparrow$ & Normal & $\uparrow$ \\
Respiratory response to hypoxia & $\uparrow$ & $\uparrow$ & Rescued \\
Lifespan & $\downarrow$ & $\downarrow$ & Rescued
\end{tabular}

In this table, an up arrow indicates increased amount of a particular phenotype, and a down arrow indicates a decreased amount. For example, weight was increased in NULL animals, decreased in CKO animals, and increased in Rescue animals. The phenotypes that are rescued (i.e., indistinguishable from WT) in the regional rescue experiment are listed as "rescued." ND indicates not done.

a fraction of cells in the hindbrain have been induced to express MeCP2 (Fig. 6D), RESCUE animals showed a substantial improvement in survival $(p<0.001$ ) (Fig. 7A). Only five animals died or became moribund and were killed (out of an initial cohort of 16 animals), with the remainder of the animals surviving $>45$ weeks, at which point some animals were killed for tissue collection.

In addition to rescuing the early lethality, restoring $\mathrm{MeCP} 2$ in this region also significantly reversed other physiological changes. In this genetic background, the NON-RESCUE animals were slightly underweight, whereas restoring MeCP2 function caused the RESCUE animals to become markedly obese (Fig. 7B), a phenotype consistent with the lack of MeCP2 within the forebrain (Fyffe et al., 2008). The NON-RESCUE animals had the same decreased heart rate seen in the NULL animals (Fig. 7C), whereas the RESCUE animals had a heart rate rescued to a WT level. However, no apparent differences in blood oxygenation existed between the genotypes (Fig. 7D).

Mimicking the NULL animals (Fig. 5A), both the NONRESCUE and the RESCUE animals showed increased baseline breathing (Fig. $5 \mathrm{~K}$ ). Thus, restoring MeCP2 function within the hindbrain did not rescue this baseline breathing abnormality. In contrast, whereas the NON-RESCUE animals showed the same relative increased breathing rate in response to hypoxia (Fig. $5 K$ ) seen in the NULL animals (Fig. 5A), the RESCUE animals showed a rescue of the breathing rate during hypoxia similar to that in WT controls (Fig. $5 \mathrm{~K}$ ). Additionally, tidal volume was generally unaffected by genotype during baseline unless normalized to body weight, in which case body weight phenotypes had a large impact on the normalized values (Fig. $5 L, M$ ). Similarly, during hypoxia, the tidal volume of RESCUE animals was not significantly different from WT animals, although NON-RESCUE animals possessed a tidal volume slightly lower than WT littermates; normalization to body weight caused the RESCUE animals to exhibit a decreased normalized tidal volume relative to littermates largely impacted by the obesity of the animals (Fig. $5 L, M$ ). Inspiratory time was unaffected by genotype during either baseline or hypoxic conditions. In contrast, the increased breathing rate phenotypes were driven by decreased expiratory times similar to the effects seen in NULL and CKO animals.

Cumulatively, these results indicate that MeCP2 function within a fraction of cells in the HoxB1 lineage is indeed sufficient to restore normal lifespan and to rescue some of the autonomic abnormalities seen in the NULL animals. Importantly, the continued presence of some observed physiological abnormalities such as abnormal baseline breathing in animals with improved survival indicates that these abnormalities are not the sole and direct cause of death.

\section{Discussion}

Traditionally, understanding the role of a specific gene product in a particular cell population, or region, of the brain relies on cellor tissue-specific deletion of the gene through a conditional knock-out approach. This conditional approach has been successfully used to understand the cell-autonomous role of MeCP2 (Fyffe et al., 2008; Adachi et al., 2009; Samaco et al., 2009; Chao et al., 2010) and has been invaluable toward determining the requirement for a gene's function in a specific cell population or region. However, in the context of distributed neural circuits coordinating behavioral and physiological processes, removing a gene from any single component of the circuit may completely disrupt the overall circuit's function. There may be many possible ways to disrupt the function of the circuit by removing the gene of interest from any number of cellular populations within that circuit. On the other hand, the ability to rescue a particular phenotype by restoring $\mathrm{MeCP} 2$ function within a select region or group of cells demonstrates conclusively that the entire neural circuit function has been restored. In the case of MeCP2 dysfunction, this has important clinical implications: the identification of an anatomically restricted region in which restoration of $\mathrm{MeCP} 2$ function causes dramatic changes in clinically relevant phenotypes implies that specific therapy, such as gene therapy, targeted toward this restricted anatomic area may have dramatic effects on clinical features.

$\mathrm{MeCP} 2$ function is required in the anatomical region defined by the HoxB1 lineage for normal lifespan. Furthermore, restoring $\mathrm{MeCP} 2$ function within this same region restores lifespan, demonstrating that $\mathrm{MeCP} 2$ function is both necessary and sufficient within the HoxB1 lineage for survival. In addition to the effect on 
lifespan, several autonomic and respiratory phenotypes are dependent on $\mathrm{MeCP} 2$ function within the HoxB1 domain. In particular, loss of MeCP2 in this region contributes to a decreased heart rate as well as a greater increase in respiratory rate during an acute hypoxic challenge. There is clear complementarity in these phenotypes, as they result from loss of MeCP2 function within the HoxB1 domain and are reversed when MeCP2 function is restored within this domain (Table 1). Survival, weight, heart rate, basal hyperventilation, and hypoxic respiratory response all exhibited reciprocal phenotypes between the HoxB1 CKO and RESCUE models. The ability to identify such reciprocal phenotypes between the CKO and RESCUE animals argues strongly for a tissue-autonomous function for $\mathrm{MeCP} 2$ in the hindbrain. Furthermore, the reciprocity of the weight phenotypes observed between the CKO and RESCUE mice (loss of MeCP2 within the HoxB1 domain causing an underweight phenotype, and loss of $\mathrm{MeCP} 2$ rostral to the HoxB1 domain causing an overweight phenotype) revealed that $\mathrm{MeCP} 2$ is required within two separate anatomical domains for competing drives affecting weight.

Interestingly, some of the abnormalities observed in the NULL mice are not reproduced when MeCP2 function is removed from the HoxB1 domain and are not rescued when MeCP2 expression is restored in this domain (Table 1). This indicates that such phenotypes are clearly the result of MeCP2 dysfunction in the region outside the HoxB1 domain. Of the phenotypes not dependent on MeCP2 function within the HoxB1 domain, perhaps the most notable is the increased breathing rate observed in the NULL animals during baseline breathing. This breathing abnormality is not present in CKO animals but is present in the RESCUE animals, suggesting that the phenotype has an origin outside the HoxB1 expression domain. The fact that some of the breathing abnormalities are dependent on MeCP2 function within the HoxB1 domain (i.e., response to hypoxia), whereas other breathing abnormalities, such as the basal hyperventilation, are not, demonstrates that $\mathrm{MeCP} 2$ function is important in multiple nodes of respiratory circuitry, and that the function within these anatomically distinct regions are separable. Furthermore, the increase in breathing rate, during basal conditions or during hypoxic challenge, is largely due to a reduction of the expiratory time per breath, a result that is consistent with models of increased net activation of respiratory pattern generating preinspiratory neurons of the pre-Botzinger complex (Smith et al., 2009). Given the anatomical separation in the requirement of MeCP2 for normal baseline or hypoxic breathing rates, the requirement of $\mathrm{MeCP} 2$ rostral to the HoxB1 domain for normal baseline respiration suggests impaired signaling from nuclei rostral to the HoxB1 domain, possibly including the parabrachial nuclei and the Kölliker-Fuse nucleus, to the ventral respiratory group of the medulla (Stettner et al., 2007; Song et al., 2010). In contrast, the requirement within the HoxB1 domain for normal respiratory rate during hypoxia suggests impairments in the circuits responding to oxygen levels. One possibility is that signaling from the NTS relaying the sensation of hypoxia detected in the carotid body to the ventral respiratory group of the medulla is altered (Teppema and Dahan, 2010); specifically, deficits in GABA signaling caused by loss of MeCP2 (Medrihan et al., 2008; Chao et al., 2010) may underlie the apparently impaired hypoxic ventilatory decline caused by loss of MeCP2 in the hindbrain, presenting as relative hyperventilation during an acute hypoxic challenge (Teppema and Dahan, 2010). Alternatively, because the carotid body itself is targeted in the $\mathrm{CKO}$ and RESCUE animals by HoxB $1^{\text {Cre }}$, direct involvement of this oxygen sensor is also a possibility.
Additionally, the origin of the abnormal response to hypoxia within the HoxB1 domain is particularly important because it has been proposed that early exposure to hypoxic stress may be an inciting feature in the development of the disrupted breathing in the mouse models as well as in girls with RTT (Voituron et al., 2009). However, the occurrence of the abnormal basal breathing rate in the RESCUE animals despite a normalized respiratory rate during hypoxia suggests that basal hyperventilation is not secondary to the chemosensory deficits. It remains to be seen whether the chemosensory deficits precede other respiratory deficits not explored in this study, or more specifically whether exposure to hypoxic stress plays a causal role in the progression of other autonomic deficits.

A surprising finding of this study is that restoring $\mathrm{MeCP} 2$ protein to only $50 \%$ of the cells within the hindbrain results in rescue of lifespan and other autonomic and breathing abnormalities. Detailed stereological quantification of MeCP2 expression across several regions of the hindbrain suggests that the approximate $50 \%$ efficiency of rescue was uniform throughout the HoxB1 domain. Similar phenotypic and histological characterization using more regionally restricted Cre lines that subdivide the HoxB1 domain are likely to refine our understanding of the anatomical requirements of $\mathrm{MeCP} 2$ function. However, the apparent uniformity of the modest efficiency of rescue suggests that restoring MeCP2 function in some cells has non-cellautonomous effects. Restoring $\mathrm{MeCP} 2$ may result in production of secreted factors such as growth factors that improve the function of nearby cells. On the other hand, the effect may be at the circuit level, where only a fraction of the cells within a circuit that express MeCP2 are capable of restoring the entire circuit's function. These possibilities are not mutually exclusive, and the overall positive effect of restoring MeCP2 function in a fraction of cells in a region may be due to both possibilities. However, there is a requirement to reestablish $\mathrm{MeCP} 2$ in particular anatomic regions to induce such a dramatic effect, because restoring $\mathrm{MeCP} 2$ function solely within the forebrain does not rescue lifespan (Alvarez-Saavedra et al., 2007). In the context of developing therapies to treat RTT, aside from the technical challenge of effectively restoring MeCP2 function in specific cells within the human brain, an additional challenge will be to determine exactly which cellular populations have critical requirements for MeCP2 function to modulate specific phenotypes. The work outlined here provides a methodology for identifying these cellular populations, which then could be useful in the development of novel regionally targeted therapies.

\section{References}

Adachi M, Autry AE, Covington HE 3rd, Monteggia LM (2009) MeCP2mediated transcription repression in the basolateral amygdala may underlie heightened anxiety in a mouse model of Rett syndrome. J Neurosci 29:4218-4227.

Alvarez-Saavedra M, Sáez MA, Kang D, Zoghbi HY, Young JI (2007) Cellspecific expression of wild-type MeCP2 in mouse models of Rett syndrome yields insight about pathogenesis. Hum Mol Genet 16:2315-2325.

Amir RE, Van den Veyver IB, Wan M, Tran CQ, Francke U, Zoghbi HY (1999) Rett syndrome is caused by mutations in X-linked MECP2, encoding methyl-CpG-binding protein 2. Nat Genet 23:185-188.

Arenkiel BR, Gaufo GO, Capecchi MR (2003) Hoxbl neural crest preferentially form glia of the PNS. Dev Dyn 227:379-386.

Chao HT, Chen H, Samaco RC, Xue M, Chahrour M, Yoo J, Neul JL, Gong S, Lu HC, Heintz N, Ekker M, Rubenstein JL, Noebels JL, Rosenmund C, Zoghbi HY (2010) Dysfunction in GABA signalling mediates autismlike stereotypies and Rett syndrome phenotypes. Nature 468:263-269.

Chen RZ, Akbarian S, Tudor M, Jaenisch R (2001) Deficiency of methylCpG binding protein-2 in CNS neurons results in a Rett-like phenotype in mice. Nat Genet 27:327-331. 
Finley JC, Maderdrut JL, Roger LJ, Petrusz P (1981) The immunocytochemical localization of somatostatin-containing neurons in the rat central nervous system. Neuroscience 6:2173-2192.

Fyffe SL, Neul JL, Samaco RC, Chao HT, Ben-Shachar S, Moretti P, McGill BE, Goulding EH, Sullivan E, Tecott LH, Zoghbi HY (2008) Deletion of Mecp2 in Sim1-expressing neurons reveals a critical role for MeCP2 in feeding behavior, aggression, and the response to stress. Neuron 59: 947-958.

Glaze DG, Percy AK, Skinner S, Motil KJ, Neul JL, Barrish JO, Lane JB, Geerts SP, Annese F, Graham J, McNair L, Lee HS (2010) Epilepsy and the natural history of Rett syndrome. Neurology 74:909-912.

Guideri F, Acampa M, Hayek G, Zappella M, Di Perri T (1999) Reduced heart rate variability in patients affected with Rett syndrome. A possible explanation for sudden death. Neuropediatrics 30:146-148.

Guy J, Hendrich B, Holmes M, Martin JE, Bird A (2001) A mouse Mecp2null mutation causes neurological symptoms that mimic Rett syndrome. Nat Genet 27:322-326.

Guy J, Gan J, Selfridge J, Cobb S, Bird A (2007) Reversal of neurological defects in a mouse model of Rett syndrome. Science 315:1143-1147.

Hippenmeyer S, Vrieseling E, Sigrist M, Portmann T, Laengle C, Ladle DR, Arber S (2005) A developmental switch in the response of DRG neurons to ETS transcription factor signaling. PLoS Biol 3:e159.

Julu PO, Kerr AM, Apartopoulos F, Al-Rawas S, Engerström IW, Engerström L, Jamal GA, Hansen S (2001) Characterisation of breathing and associated central autonomic dysfunction in the Rett disorder. Arch Dis Child 85:29-37.

Kankirawatana P, Leonard H, Ellaway C, Scurlock J, Mansour A, Makris CM, Dure LS 4th, Friez M, Lane J, Kiraly-Borri C, Fabian V, Davis M, Jackson J, Christodoulou J, Kaufmann WE, Ravine D, Percy AK (2006) Early progressive encephalopathy in boys and MECP2 mutations. Neurology 67:164-166.

Kerr AM, Armstrong DD, Prescott RJ, Doyle D, Kearney DL (1997) Rett syndrome: analysis of deaths in the British survey. Eur Child Adolesc Psychiatry 6:71-74.

Lazarenko RM, Milner TA, Depuy SD, Stornetta RL, West GH, Kievits JA, Bayliss DA, Guyenet PG (2009) Acid sensitivity and ultrastructure of the retrotrapezoid nucleus in Phox2b-EGFP transgenic mice. J Comp Neurol 517:69-86.

Madan N, Levine M, Pourmoghadam K, Sokoloski M (2004) Severe sinus bradycardia in a patient with Rett syndrome: a new cause for a pause? Pediatr Cardiol 25:53-55.

Medrihan L, Tantalaki E, Aramuni G, Sargsyan V, Dudanova I, Missler M, Zhang W (2008) Early defects of GABAergic synapses in the brain stem of a MeCP2 mouse model of Rett syndrome. J Neurophysiol 99:112-121.

Neul JL, Kaufmann WE, Glaze DG, Christodoulou J, Clarke AJ, Bahi-Buisson N, Leonard H, Bailey ME, Schanen NC, Zappella M, Renieri A, Huppke P, Percy AK (2010) Rett syndrome: revised diagnostic criteria and nomenclature. Ann Neurol 68:944-950.

Ogier M, Wang H, Hong E, Wang Q, Greenberg ME, Katz DM (2007) Brain-derived neurotrophic factor expression and respiratory function improve after ampakine treatment in a mouse model of Rett syndrome. J Neurosci 27:10912-10917.

Paxinos G, Franklin K (2001) The mouse brain in stereotaxic coordinates, Ed 2. San Diego: Elsevier.

Pratte M, Panayotis N, Ghata A, Villard L, Roux JC (2011) Progressive motor and respiratory metabolism deficits in post-weaning Mecp2-null male mice. Behav Brain Res 216:313-320.

Rasband WS (1997-2011) ImageJ. Bethesda, MD: U.S. National Institutes of Health.

Rohdin M, Fernell E, Eriksson M, Albåge M, Lagercrantz H, Katz-Salamon M (2007) Disturbances in cardiorespiratory function during day and night in Rett syndrome. Pediatr Neurol 37:338-344.

Rose MF, Ren J, Ahmad KA, Chao HT, Klisch TJ, Flora A, Greer JJ, Zoghbi HY
(2009) Math1 is essential for the development of hindbrain neurons critical for perinatal breathing. Neuron 64:341-354.

Roux JC, Dura E, Villard L (2008) Tyrosine hydroxylase deficit in the chemoafferent and the sympathoadrenergic pathways of the Mecp2 deficient mouse. Neurosci Lett 447:82-86.

Samaco RC, Fryer JD, Ren J, Fyffe S, Chao HT, Sun Y, Greer JJ, Zoghbi HY, Neul JL (2008) A partial loss of function allele of methyl-CpG-binding protein 2 predicts a human neurodevelopmental syndrome. Hum Mol Genet 17:1718-1727.

Samaco RC, Mandel-Brehm C, Chao HT, Ward CS, Fyffe-Maricich SL, Ren J, Hyland K, Thaller C, Maricich SM, Humphreys P, Greer JJ, Percy A, Glaze DG, Zoghbi HY, Neul JL (2009) Loss of MeCP2 in aminergic neurons causes cell-autonomous defects in neurotransmitter synthesis and specific behavioral abnormalities. Proc Natl Acad Sci U S A 106: $21966-21971$.

Schüle B, Armstrong DD, Vogel H, Oviedo A, Francke U (2008) Severe congenital encephalopathy caused by MECP2 null mutations in males: central hypoxia and reduced neuronal dendritic structure. Clin Genet 74:116-126.

Shahbazian M, Young J, Yuva-Paylor L, Spencer C, Antalffy B, Noebels J, Armstrong D, Paylor R, Zoghbi H (2002) Mice with truncated MeCP2 recapitulate many Rett syndrome features and display hyperacetylation of histone H3. Neuron 35:243-254.

Smith JC, Abdala AP, Rybak IA, Paton JF (2009) Structural and functional architecture of respiratory networks in the mammalian brainstem. Philos Trans R Soc Lond B Biol Sci 364:2577-2587.

Song G, Tin C, Poon CS (2010) Bilateral lesions of pontine Kolliker-Fuse nuclei provoke apnea instead of apneusis in anesthetized adult rats. Adv Exp Med Biol 669:185-188.

Soriano P (1999) Generalized lacZ expression with the ROSA26 Cre reporter strain. Nat Genet 21:70-71.

Stettner GM, Huppke P, Brendel C, Richter DW, Gärtner J, Dutschmann M (2007) Breathing dysfunctions associated with impaired control of postinspiratory activity in Mecp2-/y knockout mice. J Physiol 579:863-876.

Stornetta RL, Rosin DL, Wang H, Sevigny CP, Weston MC, Guyenet PG (2003) A group of glutamatergic interneurons expressing high levels of both neurokinin- 1 receptors and somatostatin identifies the region of the pre-Botzinger complex. J Comp Neurol 455:499-512.

Su H, Mills AA, Wang X, Bradley A (2002) A targeted X-linked CMV-Cre line. Genesis 32:187-188.

Teppema LJ, Dahan A (2010) The ventilatory response to hypoxia in mammals: mechanisms, measurement, and analysis. Physiol Rev 90:675-754.

Viemari JC, Roux JC, Tryba AK, Saywell V, Burnet H, Peña F, Zanella S, Bévengut M, Barthelemy-Requin M, Herzing LB, Moncla A, Mancini J, Ramirez JM, Villard L, Hilaire G (2005) Mecp2 deficiency disrupts norepinephrine and respiratory systems in mice. J Neurosci 25:11521-11530.

Voituron N, Zanella S, Menuet C, Dutschmann M, Hilaire G (2009) Early breathing defects after moderate hypoxia or hypercapnia in a mouse model of Rett syndrome. Respir Physiol Neurobiol 168:109-118.

Weese-Mayer DE, Lieske SP, Boothby CM, Kenny AS, Bennett HL, Silvestri JM, Ramirez JM (2006) Autonomic nervous system dysregulation: breathing and heart rate perturbation during wakefulness in young girls with Rett syndrome. Pediatr Res 60:443-449.

West MJ, Slomianka L, Gundersen HJ (1991) Unbiased stereological estimation of the total number of neurons in the subdivisions of the rat hippocampus using the optical fractionator. Anat Rec 231:482-497.

Young JI, Zoghbi HY (2004) X-chromosome inactivation patterns are unbalanced and affect the phenotypic outcome in a mouse model of Rett syndrome. Am J Hum Genet 74:511-520.

Zanella S, Mebarek S, Lajard AM, Picard N, Dutschmann M, Hilaire G (2008) Oral treatment with desipramine improves breathing and life span in Rett syndrome mouse model. Respir Physiol Neurobiol 160:116 121. 